\title{
THE TIME TO RUIN IN SOME ADDITIVE RISK MODELS WITH RANDOM PREMIUM RATES
}

\author{
MARTIN JACOBSEN, ${ }^{*}$ University of Copenhagen
}

\begin{abstract}
The risk processes considered in this paper are generated by an underlying Markov process with a regenerative structure and an independent sequence of independent and identically distributed claims. Between the arrivals of claims the process increases at a rate which is a nonnegative function of the present value of the Markov process. The intensity for a claim to occur is another nonnegative function of the value of the Markov process. The claim arrival times are the regeneration times for the Markov process. Two-sided claims are allowed, but the distribution of the positive claims is assumed to have a Laplace transform that is a rational function. The main results describe the joint Laplace transform of the time at ruin and the deficit at ruin. The method used consists in finding partial eigenfunctions for the generator of the joint process consisting of the Markov process and the accumulated claims process, a joint process which is also Markov. These partial eigenfunctions are then used to find a martingale that directly leads to an expression for the desired Laplace transform. In the final section, three examples are given involving different types of the underlying Markov process.
\end{abstract}

Keywords: Time to ruin; deficit at ruin; partial eigenfunction; martingale; optional sampling; Cramér-Lundberg equation; Rouché's theorem

2010 Mathematics Subject Classification: Primary 60K20

Secondary 60G40; 60G44; 60J35

\section{Introduction}

The class of risk processes $X=\left(X_{t}\right)_{t \geq 0}$ considered in this paper have the form

$$
X_{t}=x_{0}+\int_{0}^{t} \beta\left(Y_{s}\right) \mathrm{d} s-\sum_{n=1}^{N_{t}} U_{n}
$$

with $Y$ some Markov process, $N$ the counting process counting the number of claims incurred over time, and claims $U_{n}$ that are independent and identically distributed (i.i.d.). Thus, the random premium rate at time $t$ is $\beta\left(Y_{t}\right)$ and it is assumed that the function $\beta$ is greater than or equal to 0 . The precise form of the model is described in Section 2, but it is important already at this stage to emphasise that $X$ is constructed from $Y$ and the accumulated claims process: in the literature it is more standard to study models with random premium rates depending on the value of the risk process,

$$
X_{t}=x_{0}+\int_{0}^{t} \beta\left(X_{s}\right) \mathrm{d} s-\sum_{n=1}^{N_{t}} U_{n}
$$

Received 5 October 2009; revision received 3 May 2012.

* Postal address: Department of Mathematical Sciences, University of Copenhagen, 5, Universitetsparken, DK-2100

Copenhagen Ø, Denmark. Email address: martin@math.ku.dk 
i.e. $X$ is determined as the solution to this stochastic differential equation. A survey of models of this type can be found in [11]. There does not however appear to be many examples of processes of the form (1.1) with $Y$ not determined exclusively by $X$, one exception being $\mathrm{Wu}$ and Wei [13].

The present paper is a natural continuation of Jacobsen [5], [6]. The latter is particularly relevant and the main reference for this paper, dealing with a model that includes the special case of (1.1) when $Y$ is a certain type of Markov chain on a finite state space. Our aim is to determine the structure of the joint Laplace transform of the time to ruin and the deficit at ruin.

Model (1.2) with $N$ a Poisson process and the claims i.i.d. is discussed in Chapter 7 of [2]. Jasiulewicz [9] considered model (1.2) with $N$ a Cox process with a stochastic intensity process given as a function of a finite state space Markov chain. Wu and Wei [13] also assumed $N$ to be a Cox process with the same kind of intensity process, but they considered $X$ to be of the form (1.1) with $Y$ this intensity process. Ganesh et al. [4] studied a variation of (1.2) with the accumulated claims process of Poisson shot noise form. Møller [10] considered a general model of the form (1.2) using a marked point process to describe the occurrence and size of claims.

A rather sophisticated model, somewhat related to (1.2) in the sense that $X$ solves a stochastic differential equation, is that considered by Paulsen and Gjessing [12]. The risk process $X$ is then Markov. Their paper is also relevant because in Theorem 2.1 therein they showed that, e.g. the Laplace transform of the time to ruin may be found by determining what we refer to as a 'partial eigenfunction' for the generator of $X$, the technique used in this paper as well as in [5], [6], and [8]. By a 'partial eigenfunction' we mean a real-valued function that satisfies the basic eigenfunction identity only on a subset of the set on which the function is defined. This concept is different from that of 'partial eigenfunction expansions' encountered, e.g. in Fourier analysis.

Most of the literature focuses on determining the probability of ruin within a given time interval $[0, T]$, e.g. how it can be found (as a function of the initial state $x_{0}$ and $T$ ) by solving functional equations of differential and/or integral type. Exact results are given in [2, Corollary 7.1.8] for the case of exponential claims and Poisson arrivals. In [12] the authors for some special cases of their model were able to find explicit forms of the ruin probability and the Laplace transform of the time to ruin: some of their examples allow a distribution for the claims that is not exponential but either a mixture of two exponentials or a convolution of two exponentials. For model (1.2) with $\beta(x)=\beta x$, Jacobsen and Jensen [8] in particular determined the Laplace transform of the time to $X$ crossing below a low level $l$ (for the time to ruin, $l=0$ ) when assuming that the density of the claims distribution is a linear combination of exponentials.

In order to get reasonably explicit results, it seems necessary that there is some Markov structure: in [2, Chapter 7] and [12], the risk process itself is Markov, in both [9] and [13] $X$ need not be Markov, but $(X, Y)$ is (with $Y$ the intensity for the Cox process). For our model of the form (1.1), it is certainly essential that $(X, Y)$ is Markov. It is also important for us that the distribution of the downward claims (we allow for two sided $U_{n}$ ) is of a special form, viz. with a Laplace transform that is a rational function.

The problem of determining the partial eigenfunctions for the $(X, Y)$-generator needed for determining the Laplace transform of the time to ruin is not simple, but the structure of our model allows us to separate the two variables $x$ and $y$ (see (3.25) below for the analytic form of the partial eigenfunctions), reducing the problem to that of finding the solutions to a CramérLundberg-type equation (see (3.6) below) and solving a functional equation (see (3.5) below) which does not involve $(X, Y)$ but only what we shall call a generic Markov process $Y^{\circ}$ from 
which $Y$ is obtained by imposing a regenerative structure that is essential for the analysis presented below.

Our model is complicated in the sense that although $(X, Y)$ is Markov, $X$ itself in general is not. It is simple in the sense that $X$ is additive, i.e. to start the process at a new level $x_{1}$, just add the constant $x_{1}-x_{0}$ on both sides of (1.1). By contrast, when $X$ is Markov, model (1.2) is simple in a Markovian sense, but complicated in the sense that typically it is not additive.

The discussion above has focused on models where the claims $U_{n}$ are i.i.d. Albrecher and Boxma [1] discussed a model of the form (1.1) with $\beta(y)$ a constant $c>0$, but where the jumps of $N$ and the distribution of the claims are governed by a Markov chain on a finite state space. It is quite possible that the ruin problem for this type of model can also be solved using partial eigenfunctions; see the discussion in Remark 3.3 below where some of the difficulties that arise are mentioned.

\section{The model and the problem}

The object of study in this paper is a time-homogeneous Markov process $(X, Y)=$ $\left(X_{t}, Y_{t}\right)_{t \geq 0}$ with $X$ real-valued and $Y$ itself Markov with values in a state space $(E, \mathcal{E})$ that we shall assume is a Polish space $E$ equipped with the Borel $\sigma$-algebra $\mathscr{E}$. One way of constructing $(X, Y)$ corresponding to an arbitrary initial state $\left(X_{0}, Y_{0}\right) \equiv\left(x_{0}, y_{0}\right) \in \mathbb{R} \times E$ is as follows. Suppose that a generic time-homogeneous Markov process $Y^{\circ}=\left(Y_{t}^{\circ}\right)_{t \geq 0}$ with state space $E$ is given, and assume that $Y^{\circ}$ is càdlàg and strong Markov; suppose also that a probability measure $A$, the reset distribution, on $(E, \mathcal{E})$ and a continuous function $\lambda$ from $E$ to $\mathbb{R}_{0}=[0, \infty)$ are given. Consider a sequence $\left(Y^{(n)}\right)_{n \geq 2}$ of i.i.d. copies of $Y^{\circ}$ with initial distribution $A$ and a copy $Y^{(1)}$ of $Y^{\circ}$ with fixed initial state $y_{0}$, independent of the sequence $\left(Y^{(n)}\right)_{n \geq 2}$. Next, kill the $Y^{(n)}$ for $n \geq 1$ independently of each other at random times $T^{(n)}$ where the conditional distribution of $T^{(n)}$ given $Y^{(n)}$ is determined as

$$
\mathbb{P}\left(T^{(n)}>t \mid Y^{(n)}\right)=\exp \left(-\int_{0}^{t} \lambda\left(Y_{s}^{(n)}\right) \mathrm{d} s\right) .
$$

Define $\tau_{0} \equiv 0$ and

$$
\tau_{n}=T^{(1)}+\cdots+T^{(n)} \text { for } n \geq 1,
$$

and then construct the càdlàg component $Y$ of the desired process $(X, Y)$ by gluing the killed $Y^{(n)}$ together:

$$
Y_{t}=Y_{t-\tau_{n-1}}^{(n)}, \quad n \geq 1, \tau_{n-1} \leq t<\tau_{n} .
$$

To finally construct the second component $X$ of $(X, Y)$, let the counting process $N=\left(N_{t}\right)_{t \geq 0}$ be given by

$$
N_{t}=\sum_{n=1}^{\infty} \mathbf{1}_{\left(\tau_{n} \leq t\right)}
$$

with $N_{0} \equiv 0$ and introduce a sequence $\left(U_{n}\right)_{n \geq 1}$ of i.i.d. $\mathbb{R}$-valued random variables with distribution $F$, independent of $Y$ and the $T_{n}$ and satisfying

$$
\mathbb{P}\left(U_{n}>0\right)>0 .
$$

Finally, let $\beta: E \rightarrow \mathbb{R}_{0}$ be a given nonnegative, continuous function. Then define, corresponding to the given initial state $x_{0}$ for $X$,

$$
X_{t}=x_{0}+\int_{0}^{t} \beta\left(Y_{s}\right) \mathrm{d} s-\bar{U}_{t},
$$


where

$$
\bar{U}_{t}=\sum_{n=1}^{N_{t}} U_{n}
$$

Thus, thinking of the $\tau_{n}$ as the claim arrival times with $U_{n}$ the size of the $n$th claim, we see that the claims arrive according to a delayed renewal process with interarrival times $\tau_{n}-\tau_{n-1}=T^{(n)}$ for $n \geq 2$ i.i.d. with

$$
\mathbb{P}\left(T^{(n)}>t\right)=\mathrm{E}_{A}\left(\exp \left(-\int_{0}^{t} \lambda\left(Y_{s}^{\circ}\right) \mathrm{d} s\right)\right) .
$$

Also, between claims, $X$ increases at the rate $\beta\left(Y_{s}\right)$. (Formally, one could allow for arbitrary $\mathbb{R}$-valued functions $\beta$, but in the main result, Theorem 3.1 below, it is important that $\beta \geq 0$ in order to allow only for 'ruin by jump'.)

Notation. We write $\mathbb{P}$ and $\mathbb{E}$ for the probability measure and expectation when referring to the space where all the random variables involved in the construction of $(X, Y)$ are defined. When referring to the generic process $Y^{\circ}$, we write $\mathrm{P}$ and $\mathrm{E}$. An index refers to how the process(es) is started, either using a fixed initial state (such as $y_{0}$ or $\left(x_{0}, y_{0}\right)$ ) or using a distribution for the initial random variable $\left(Y_{0}^{\circ}\right.$ or $\left(X_{0}, Y_{0}\right)$ ). If no index is specified, it signifies that the probability or expectation is the same for all initial distributions. Similarly, writing, e.g. $\mathbb{P}_{y}$ signifies that the probability in question is the same for all initial distributions of $X$ and with $Y_{0} \equiv y$.

It was assumed above that the functions $\lambda$ and $\beta$ are both greater than or equal to 0 and continuous. We shall further assume for convenience that the nonnegative càdlàg processes $\lambda\left(Y^{\circ}\right)$ and $\beta\left(Y^{\circ}\right)$ both have finite left limits everywhere, so that in particular, for all $t \geq 0$ and all $y \in E$,

$$
\mathrm{P}_{y}\left(\int_{0}^{t} \lambda\left(Y_{s}^{\circ}\right) \mathrm{d} s<\infty\right)=\mathrm{P}_{y}\left(\int_{0}^{t} \beta\left(Y_{s}^{\circ}\right) \mathrm{d} s<\infty\right)=1 .
$$

Then $X$ defined by (2.3) is everywhere finite and all $T^{(n)}>0, \mathbb{P}_{x, y}$-almost surely $\left(\mathbb{P}_{x, y}\right.$-a.s.) for all $x, y$. In order that ruin be at all possible, it is necessary that $T^{(1)}<\infty$ can occur; hence, we also assume that, for some $y \in E$,

$$
\mathrm{P}_{y}\left(\int_{0}^{\infty} \lambda\left(Y_{s}^{\circ}\right) \mathrm{d} s>0\right)>0,
$$

a condition satisfied for a particular $y$ if $\lambda(y)>0$. As regards the total number of jumps, the most interesting case is when $N$ and $X$ have infinitely many jumps as will happen if, e.g.

$$
\mathrm{P}_{y}\left(\int_{0}^{\infty} \lambda\left(Y_{s}^{\circ}\right) \mathrm{d} s=\infty\right)=1
$$

for all $y$. We shall however not assume this, and so will allow for $N$ and $X$ to have only finitely many jumps; cf. Example 3.1 below. Note that if all $T^{(n)}$ are finite a.s., the fact that they are i.i.d. for $n \geq 2$ ensures that $\tau_{n} \uparrow \infty$ a.s., i.e. the infinitely many jumps will not accumulate in finite time.

It is often assumed that a jump in $N$ forces a jump in $X$, i.e. $\mathbb{P}\left(U_{n} \neq 0\right)=1$. However, this assumption is not necessary here, so at this stage the distribution $F$ of the $U_{n}$ is an arbitrary probability on $\mathbb{R}$ subject to (2.2).

Three special choices of $Y^{\circ}$ should be mentioned. (i) $Y^{\circ}$ is a time-homogeneous Markov chain on a finite state space; see [5] and [6] with the model in [5] allowing for one-sided jumps 
only, and the model in [6] being more general than (2.3) in the sense that $\beta$ is allowed to be negative and $X$ may contain an additional Brownian term $\sigma\left(Y_{t}\right) \mathrm{d} B_{t}$ so that ruin by creeping ( $X$ may slide across 0 between jumps) is possible. (ii) $Y^{\circ}$ is a time-homogeneous (one-dimensional) diffusion - this is the motivating example for this research; see the specific case in Example 4.3 below. (iii) $Y^{\circ}$ is purely deterministic in the sense that only the initial value $Y_{0}^{\circ}$ is random. This is the simplest case where it is possible to use Theorem 3.1 below for explicit analytical results; see Example 4.2 below.

Example 2.1. The same model for $X$ may have many representations of the form (2.3). The standard risk model with Poisson arrivals of claims and constant premium rate $\beta$ may thus be obtained by using any generic process $Y^{\circ}$, taking $\beta(y) \equiv \beta$ and $\lambda(y) \equiv \lambda>0$ in the general setup above. It should be noted that then in Theorem 3.1 below, the solutions to the critical equations (3.5) and (3.6) do not depend on $Y^{\circ}$ (or $Y$ ) either: the unique bounded solution to (3.5) is always the constant function

$$
c_{\gamma}(y) \equiv \frac{\lambda}{\gamma \beta-\lambda-\theta}
$$

and (3.6) then reduces to

$$
R_{+}(\gamma)\left(1+\left(1-p_{U}\right) L_{-}(\gamma) \frac{\lambda}{\gamma \beta-\lambda-\theta}\right)=-p_{U} P_{+}(\gamma) \frac{\lambda}{\gamma \beta-\lambda-\theta} .
$$

A final comment on the structure of the model is that it is additive in the sense that, for any $x \in \mathbb{R}, y \in E$, and $w \in \mathbb{R}$, the $\mathbb{P}_{x+w, y}$-law of $(X, Y)$ is the same as the $\mathbb{P}_{x, y}$-law of the process $(X+w, Y)$.

The analysis to be done below is in part based on applications of Itô's formula. To facilitate this, let $\mathcal{A}_{Y^{\circ}}$ denote the infinitesimal generator for $Y^{\circ}$ with a domain $\mathscr{D}_{Y}$ 。 consisting of all bounded and continuous functions $g: E \rightarrow \mathbb{R}$ such that $\mathcal{A}_{Y^{\circ}} g: E \rightarrow \mathbb{R}$ is bounded and continuous with

$$
M_{t}^{g}=g\left(Y_{t}^{\circ}\right)-g(y)-\int_{0}^{t} \mathcal{A}_{Y \circ} g\left(Y_{s}^{\circ}\right) \mathrm{d} s
$$

a true mean-zero martingale (under any $\mathrm{P}_{y}$ using the filtration generated by $Y^{\circ}$ ). The infinitesimal generator $\mathcal{A}_{Y}$ for $Y$ then has the form

$$
\mathcal{A}_{Y} g(y)=\mathcal{A}_{Y^{\circ}} g(y)+\lambda(y) \int_{E} A(\mathrm{~d} v)(g(v)-g(y))
$$

and, for the generator $\mathcal{A}_{X, Y}$ for $(X, Y)$, we obtain the form

$$
\begin{aligned}
\mathcal{A}_{X, Y} f(x, y)= & \mathcal{A}_{Y \circ} f(x, \cdot)(y)+\beta(y) \frac{\partial}{\partial x} f(x, y) \\
& +\lambda(y) \int_{\mathbb{R}} F(\mathrm{~d} u) \int_{E} A(\mathrm{~d} v)(f(x-u, v)-f(x, y))
\end{aligned}
$$

for suitably smooth bounded functions $f$.

Remark 2.1. Suppose that $g \in \mathscr{D}_{Y^{\circ}}$. The martingale $M^{g}$ is then uniformly bounded on any finite interval $\left[0, t_{0}\right]$ and is therefore $L^{2}$-bounded on any finite interval. Consequently, for the quadratic variation of $M^{g}$, it holds that $\mathrm{E}_{y}\left[M^{g}\right]_{t}<\infty$ for all $t$ and, therefore, the stochastic integral $\int_{0}^{t} Z_{s}^{\circ} \mathrm{d} M_{s}^{g}$, with $Z^{\circ}$ a uniformly bounded predictable process, also defines a true martingale ( $L^{2}$-bounded on all finite intervals). This observation will be used below. 
In the first main result of the paper, Theorem 3.1, we determine the joint distribution of the time $\tau$ to ruin and the deficit $Z$ at ruin, where

$$
\tau=\inf \left\{t \geq 0: X_{t}<0\right\}, \quad Z=-X_{\tau},
$$

with $Z$ defined only on the set $(\tau<\infty)$. More precisely, we shall, for $x \geq 0$ and $y \in E$, find the joint Laplace transform

$$
\mathbb{E}_{x, y} \mathrm{e}^{-\theta \tau-\zeta Z}, \quad \theta>0, \zeta \geq 0
$$

In the second main result, Theorem 3.2, we determine the marginal Laplace transform

$$
\mathbb{E}_{x, y}\left[\mathrm{e}^{-\zeta Z} ; \tau<\infty\right], \quad \zeta \geq 0
$$

obtainable of course from (2.11) by letting $\theta \downarrow 0$. For $\zeta=0$, (2.12) yields the probability of ruin $\mathbb{P}_{x, y}(\tau<\infty)$.

As in [6], the main idea is to look for partial eigenfunctions $h$ (depending on $(\theta, \zeta)$ ) for the generator $\mathcal{A}_{X, Y}$ (see (3.24) below), since then (2.11) is obtained immediately. The first main result, Theorem 3.1, reduces the problem of finding $h(x, y)$ to that of solving a functional equation involving only the argument $y$ and the generator $\mathcal{A}_{Y^{\circ}}$; see (3.5). Essentially, it is the regenerative structure of $(X, Y)$ that allows us to replace the search for a function depending on both $x$ and $y$ with the search for a function depending on $y$ alone.

Only in exceptional cases is it possible to solve the functional equation (3.5) explicitly. Some examples are presented in Section 4.

As already noted, we do not assume that (2.7) holds for all $y$. There is however a variation of this that is important for understanding whether ruin is certain or not. Call $(X, Y)$ A-jumping if (2.7) holds for $A$-almost all $y$. Then, cf. (2.4), for $n \geq 2$,

$$
\mathbb{P}_{y}\left(T^{(n)}=\infty\right)=0
$$

for all $y$, i.e. if $\tau_{1}<\infty$, the risk process $X$ will have infinitely many jumps on $(0, \infty)$. Furthermore, the renewal structure implies that the increments

$$
X_{\tau_{n}}-X_{\tau_{n-1}}=\int_{0}^{T^{(n)}} \beta\left(Y_{s}^{(n)}\right) \mathrm{d} s-U_{n}
$$

are i.i.d. for $n \geq 2$, and since we shall assume below that $\mathbb{E} U_{n}<\infty$, the expectation of these increments,

$$
\xi=\mathbb{E}_{A}\left[\int_{0}^{T^{(1)}} \beta\left(Y_{s}^{(1)}\right) \mathrm{d} s-U_{1}\right],
$$

is well defined with $-\infty<\xi \leq \infty$. Since, on $\left(\tau_{1}<\infty\right)$,

$$
\frac{1}{n} \sum_{k=2}^{n}\left(X_{\tau_{k}}-X_{\tau_{k-1}}\right)=\frac{1}{n}\left(X_{\tau_{n}}-X_{\tau_{1}}\right) \rightarrow \xi \quad \text { a.s., }
$$

it follows that if $(X, Y)$ is $A$-jumping then, given that $\tau_{1}<\infty$, if $\xi \leq 0$, ruin is certain, i.e.

$$
\mathbb{P}_{x, y}\left(\tau<\infty \mid \tau_{1}<\infty\right)=1, \quad x \geq 0, y \in E,
$$


or, since $\tau \geq \tau_{1}$ always,

$$
\mathbb{P}_{x, y}(\tau<\infty)=\mathbb{P}_{y}\left(\tau_{1}<\infty\right)=1-\mathrm{E}_{y}\left(\exp \left(-\int_{0}^{\infty} \lambda\left(Y_{s}^{\circ}\right) \mathrm{d} s\right)\right)
$$

with the ruin probability not depending on $x$.

If $(X, Y)$ is $A$-jumping and $\xi>0$, ruin is not certain with, in particular,

$$
\lim _{x \rightarrow \infty} \mathbb{P}_{x, y}(\tau<\infty)=0, \quad y \in E,
$$

as is easily seen since $X_{\tau_{n}} \rightarrow \infty$ a.s. on $\left(\tau_{1}<\infty\right)$, so $I:=\inf \left\{X_{t}: t \geq 0\right\}>-\infty$ a.s., and then (2.14) follows using the additivity of the model.

If $(X, Y)$ is $A$-jumping, we call $(X, Y) A$-recurrent if $\xi \leq 0$ and $A$-transient if $\xi>0$. If $(X, Y)$ is not $A$-jumping, we have $\mathbb{P}_{A}\left(T^{(n)}=\infty\right)>0$ for each $n \geq 2$, and since these $T^{(n)}$ are i.i.d., it follows that $X$ has only finitely many jumps on $(0, \infty)$, so ruin is not certain. In this case $I>-\infty$ a.s. and (2.14) is true.

\section{The main results}

For Theorem 3.1 below, we need the following structure on the distribution $F$ of the claim sizes $U_{n}$, which was also used in [6]: write

$$
F(\mathrm{~d} u)= \begin{cases}p_{U} F_{+}(\mathrm{d} u), & u>0, \\ \left(1-p_{U}\right) F_{-}(\mathrm{d} u), & u \leq 0,\end{cases}
$$

with $F_{+}$a probability measure on $(0, \infty)$ and $F_{-}$a probability measure on $(-\infty, 0]$. Assume that $0<p_{U} \leq 1$ so that positive claims (negative jumps for $X$ ) are always possible. If $p_{U}=1$, only positive claims occur and $F_{-}$is irrelevant. Next, introduce the Laplace transforms

$$
\begin{array}{ll}
L_{+}(v)=\mathbb{E}\left[\mathrm{e}^{-v U_{n}} \mid U_{n}>0\right]=\int_{(0, \infty)} \mathrm{e}^{-v u} F_{+}(\mathrm{d} u), & v \geq 0, \\
L_{-}(v)=\mathbb{E}\left[\mathrm{e}^{-v U_{n}} \mid U_{n} \leq 0\right]=\int_{(-\infty, 0]} \mathrm{e}^{-v u} F_{-}(\mathrm{d} u), & v \leq 0 .
\end{array}
$$

The definitions of $L_{+}$and $L_{-}$as integrals extend trivially to $\{v \in \mathbb{C}: \operatorname{Re} v \geq 0\}$ in the case of $L_{+}$and to $\mathbb{C}_{-}:=\{v \in \mathbb{C}: \operatorname{Re} v \leq 0\}$ in the case of $L_{-}$. In particular,

$$
\left|L_{+}(z)\right| \leq 1, \quad \operatorname{Re} z \geq 0 .
$$

The basic assumption we shall make concerns $L_{+}$, which is supposed to be a rational function:

$$
L_{+}(v)=\frac{P_{+}(v)}{R_{+}(v)}
$$

Here $P_{+}$and $R_{+}$are polynomials with no common roots, and $R_{+}$is of degree $m \geq 1$, conveniently standardised so that the leading term of degree $m$ has coefficient 1 . In particular, $P_{+}$is of degree less than $m$. Note that $L_{+}$has an analytic extension to all of $\mathbb{C}$ except for the $m$ roots $\rho$ of $R_{+}$, all of which satisfy $\operatorname{Re} \rho<0$.

The reader is reminded that the distributions on $\mathbb{R}_{+}$with a Laplace transform of the form (3.2) are the distributions with a Lebesgue density $f(x) \geq 0$, which is a finite linear combination of terms $x^{p} \mathrm{e}^{-\mu x}$ with $p \geq 0$ an integer and $\mu \in \mathbb{C}$ with $\operatorname{Re} \mu>0$.

If $p_{U}<1$, we shall also need to suppose that $L_{-}$has a suitable analytic extension from $\mathbb{C}_{-}$, an assumption most easily satisfied if $F_{-}$is light tailed, e.g. if $L_{-}$is also a rational function. 
The main result of this paper is the following, valid only because $\beta \geq 0$; see Remark 3.2 below.

Theorem 3.1. (i) Suppose that, for every $\theta>0$ and every $\gamma \in \mathbb{C}$ with $\operatorname{Re} \gamma<0$, the function

$$
c_{\gamma, \theta}(y)=-\mathrm{E}_{y} \int_{0}^{\infty} \lambda\left(Y_{s}^{\circ}\right) \exp \left(\int_{0}^{s}\left(\gamma \beta\left(Y_{u}^{\circ}\right)-\lambda\left(Y_{u}^{\circ}\right)-\theta\right) \mathrm{d} u\right) \mathrm{d} s
$$

defined on $E$ is continuous. Then

$$
\left|c_{\gamma, \theta}(y)\right|<1, \quad \gamma \in \mathbb{C}_{-}, y \in E,
$$

and $c_{\gamma, \theta}$ belongs to the domain $\mathscr{D}_{Y} \circ$ of the generator $\mathcal{A}_{Y} \circ$ and is the unique bounded solution to the functional equation

$$
(\gamma \beta(y)-\lambda(y)-\theta) c_{\gamma, \theta}(y)+\mathcal{A}_{Y^{\circ}} c_{\gamma, \theta}(y)=\lambda(y), \quad y \in E .
$$

(ii) In addition to the continuity of the $c_{\gamma, \theta}$, suppose that $L_{+}$is given by (3.2) with $R_{+}$ a polynomial of degree $m$. If $p_{U}<1$, suppose also that $L_{-}$is analytic in an open set containing $\mathbb{C}_{-}$. Finally, suppose that, for each $\theta>0$, the function $\gamma \mapsto \int_{E} A(\mathrm{~d} v) c_{\gamma, \theta}(v)$ for $\gamma$ with $\operatorname{Re} \gamma<0$ is analytic with an analytic extension to some open set $D=D(\theta) \supseteq \mathbb{C}_{-}$. Then the Cramér-Lundberg equation

$$
R_{+}(\gamma)\left(1+\left(1-p_{U}\right) L_{-}(\gamma) \int_{E} A(\mathrm{~d} v) c_{\gamma, \theta}(v)\right)=-p_{U} P_{+}(\gamma) \int_{E} A(\mathrm{~d} v) c_{\gamma, \theta}(v)
$$

has precisely $m \theta$-dependent solutions $\gamma_{1}(\theta), \ldots, \gamma_{m}(\theta) \in\{z \in \mathbb{C}: \operatorname{Re} z<0\}$ (counted with multiplicity) and it furthermore holds for all $\theta>0$ for which these $m$ solutions are distinct that

$$
\mathbb{E}_{x, y} \mathrm{e}^{-\theta \tau-\zeta Z}=\frac{L_{+}(\zeta) \sum_{k=1}^{m} r_{k} c_{\gamma_{k}}(\theta), \theta}{(y) \mathrm{e}^{\gamma_{k}(\theta) x}}, \quad x \geq 0, y \in E,
$$

for all $\zeta \geq 0$, where $q_{k}$ and $r_{k}$ depend on $\theta$ and $\zeta$ with

$$
\begin{aligned}
q_{k} & =\frac{P_{+}\left(\gamma_{k}(\theta)\right)}{\left(\gamma_{k}(\theta)-\zeta\right) \prod_{k^{\prime} \neq k}\left(\gamma_{k}(\theta)-\gamma_{k^{\prime}}(\theta)\right)}, \\
r_{k} & =-\frac{p_{U} q_{k}}{1+\left(1-p_{U}\right) L_{-}\left(\gamma_{k}(\theta)\right) \int_{E} A(\mathrm{~d} v) c_{\gamma_{k}(\theta), \theta}(v)} .
\end{aligned}
$$

Remark 3.1. The assumption that the functions $c_{\gamma, \theta}$ be continuous is a genuine assumption on the structure of the Markov process $Y^{\circ}$, satisfied by the processes normally studied, e.g. most diffusions, all Lévy processes, all Markov chains on a finite or countably infinite state space, and, more generally, all Markov processes for which the transition operators map the space of bounded continuous functions into itself.

Remark 3.2. Identity (3.7) is true only when assuming that $\beta \geq 0$. Without this assumption, the function $h$ determined by (3.25) below would still be a partial eigenfunction for the generator $\mathcal{A}_{X, Y}$, but instead of (3.26) below we would now only have

$$
h(x, y)=K \mathbb{E}_{x, y}\left[\mathrm{e}^{-\theta \tau-\zeta Z} ; A_{\mathrm{j}}\right]+\mathbb{E}_{x, y} \mathrm{e}^{-\theta \tau}\left[h\left(0, Y_{\tau}\right) ; A_{\mathrm{c}}\right], \quad x \geq 0, y \in E,
$$

where $A_{\mathrm{j}}$ is the set where ruin occurs by a jump by $X$ below 0 , while $A_{\mathrm{c}}$ is the set where ruin occurs by continuity (creeping). If $\beta \geq 0$, of course, $A_{\mathrm{c}}=\varnothing$, but if $\beta(y)<0$ is possible, 
the second term on the right-hand side of (3.10) cannot be ignored and much extra effort will be required to identify the joint Laplace transform for $\tau$ and $Z$. Theorem 1 of [6] essentially corresponds to assuming that $E$ is finite with $Y^{\circ}$ a Markov chain, and there the difficulty of the second term is resolved by showing that there is exactly the right number of partial eigenfunctions that allows one to set up a solvable system of linear equations in the unknowns

$$
\mathbb{E}_{x, y}\left[\mathrm{e}^{-\theta \tau-\zeta Z} ; A_{\mathrm{j}}\right] \quad \text { and } \mathbb{E}_{x, y}\left[\mathrm{e}^{-\theta \tau} h\left(0, Y_{\tau}\right) ; A_{\mathrm{c}} \cap\left(Y_{\tau}=i\right)\right], \quad i \in E_{\mathrm{c}},
$$

where $E_{\mathrm{c}}$ is the set of states $i \in E$ allowing ruin by creeping, i.e. $\beta(i)<0$. In this paper, where $E$ may well be uncountable, this approach is just out of the question.

Example 3.1. Because of (2.6), $\lambda(y) \equiv 0$ is not allowed, but Theorem 3.1 in fact applies also to this uninteresting case: ruin is impossible so, trivially, $\mathbb{E}_{x, y} \mathrm{e}^{-\theta \tau}=0$ for all $\theta>0, x \geq 0$, and $y \in E$ in agreement with the observation that $c_{\gamma, \theta}(y) \equiv 0$ is now the unique bounded solution to (3.5) so that (3.7) also gives $\mathbb{E}_{x, y} \mathrm{e}^{-\theta \tau}=0$.

For the proof of Theorem 3.1, we shall need Lemmas 3.1 and 3.2 below.

Lemma 3.1. Let $g$ belong to the domain $\mathcal{D}_{Y \circ}$ of the generator $\mathcal{A}_{Y \circ}$, see (2.9), let $\phi: \mathbb{R}_{0} \rightarrow \mathbb{R}$ be bounded with a continuous first derivative, and let $\psi:(-\infty, 0)$ be bounded and measurable. Define

$$
h(x, y)= \begin{cases}\phi(x) g(y), & x \geq 0, y \in E, \\ \psi(x), & x<0, y \in E,\end{cases}
$$

and assume that $\mathcal{A}_{X, Y} h(x, y)$ is bounded for $x \geq 0$ and $y \in E$. Then, for all $x_{0} \geq 0$ and $y_{0} \in E$, it holds for all $\theta>0$ that

$$
\mathbb{E}_{x_{0}, y_{0}} \mathrm{e}^{-\theta \tau} \psi\left(X_{\tau}\right)=\phi\left(x_{0}\right) g\left(y_{0}\right)+\mathbb{E}_{x_{0}, y_{0}} \int_{0}^{\tau} \mathrm{e}^{-\theta s}\left(\mathcal{A}_{X, Y} h\left(X_{s}, Y_{s}\right)-\theta h\left(X_{s}, Y_{s}\right)\right) \mathrm{d} s,
$$

while, for $\theta=0$, it holds for all $t \geq 0$ that

$$
\mathbb{E}_{x_{0}, y_{0}} \psi\left(X_{\tau \wedge t}\right)=\phi\left(x_{0}\right) g\left(y_{0}\right)+\mathbb{E}_{x_{0}, y_{0}} \int_{0}^{\tau \wedge t} \mathcal{A}_{X, Y} h\left(X_{s}, Y_{s}\right) \mathrm{d} s .
$$

Note. It is important that it is not required that $h$ globally belongs to the domain of $\mathcal{A}_{X, Y}$; in particular, $h$ is allowed to be discontinuous in $x$ at $x=0$. For the Lebesgue integral on the right-hand side of (3.11), only $\mathcal{A}_{X, Y} h\left(X_{s}, Y_{s}\right)$ for $s<\tau$ is required, i.e. it suffices that $\mathcal{A}_{X, Y} h(x, y)$ is well defined for $x \geq 0$ and $y \in E$. Then we can just use (2.10), which, for $h$, becomes

$$
\begin{aligned}
\mathcal{A}_{X, Y} h(x, y)= & \phi(x) \mathcal{A}_{Y \circ} g(y)+\beta(y) \phi^{\prime}(x) g(y)-\lambda(y) \phi(x) g(y) \\
& +\lambda(y) \int F(\mathrm{~d} u) \int_{E} A(\mathrm{~d} v) k(x, u, v),
\end{aligned}
$$

where

$$
k(x, u, v)=\mathbf{1}_{(x \geq u)} \phi(x-u) g(v)+\mathbf{1}_{(x<u)} \psi(x-u) .
$$

Proof of Lemma 3.1. The key step in the proof is to argue that, for all $x_{0} \geq 0, y_{0} \in E, t \geq 0$, and $n \geq 1$,

$$
\begin{aligned}
& \mathbb{E}_{x_{0}, y_{0}} \mathrm{e}^{-\theta \tau \wedge \tau_{n} \wedge t} h\left(X_{\tau \wedge \tau_{n} \wedge t}, Y_{\tau \wedge \tau_{n} \wedge t}\right) \\
&=\phi\left(x_{0}\right) g\left(y_{0}\right)+\mathbb{E}_{x_{0}, y_{0}} \int_{0}^{\tau \wedge \tau_{n} \wedge t} \mathrm{e}^{-\theta s} \mathcal{A}_{X, Y}^{\theta} h\left(X_{s}, Y_{S}\right) \mathrm{d} s,
\end{aligned}
$$

where we write $\mathcal{A}_{X, Y}^{\theta} h=\mathcal{A}_{X, Y} h-\theta h$. From (3.13), if $\theta>0$, (3.11) then follows by dominated convergence, letting first $n$ and then $t$ tend to $\infty$, where, for the latter convergence, it is essential 
that $\theta>0$. For $\theta=0$, (3.12) follows from (3.13) by just letting $n \rightarrow \infty$. Equation (3.13) is shown by induction, with the induction step from $n$ to $n+1$ using the strong Markov property for $(X, Y)$ together with the fact that $\tau$ is one of the $\tau_{n}$ and that $\tau_{n+1}$ is the time of the first jump after $\tau_{n}$ so that (3.13) for $n=1$ can be used when conditioning on $\left(X_{s}, Y_{s}\right)_{0 \leq s \leq \tau_{n}}$. For the start of the induction, i.e. the proof of (3.13) for $n=1$, the idea is to evaluate both expectations in terms of $\mathrm{E}_{y_{0}}$-expectations involving $Y^{\circ}$ only, as can be done by using the conditional distribution of $\tau_{1}$ given $X^{(1)}$, cf. (2.1) for $n=1$, together with the fact that

$$
X_{s}^{(1)}=x_{0}+\int_{0}^{s} \beta\left(Y_{u}^{(1)}\right) \mathrm{d} u
$$

has the same distribution as $x_{0}+\int_{0}^{s} \beta\left(Y_{u}^{\circ}\right) \mathrm{d} u$. By invoking decomposition (2.9), (3.13) for $n=1$ then follows in a straightforward manner.

The second lemma required for the proof of Theorem 3.1 is used implicitly in the proof of Theorem 1 of [6]; the reader is referred to that proof for further details in the cursory proof of the lemma given below.

Lemma 3.2. Let $\gamma \mapsto d(\gamma)$ be a $\mathbb{C}$-valued function, and suppose that the equation

$$
R_{+}(\gamma)\left(1+\left(1-p_{U}\right) L_{-}(\gamma) d(\gamma)\right)=-p_{U} P_{+}(\gamma) d(\gamma)
$$

is solved by $m$ distinct numbers $\gamma_{1}, \ldots, \gamma_{m} \in \mathbb{C}$ with all $\operatorname{Re} \gamma_{k}<0$ and all $\left|d\left(\gamma_{k}\right)\right| \leq 1$. Then, with

$$
\begin{aligned}
r_{k} & =\frac{R_{+}\left(\gamma_{k}\right)}{d\left(\gamma_{k}\right)\left(\gamma_{k}-\zeta\right) \prod_{k^{\prime} \neq k}\left(\gamma_{k}-\gamma_{k^{\prime}}\right)} \\
& =-\frac{p_{U} P_{+}\left(\gamma_{k}\right)}{\left(1+\left(1-p_{U}\right) L_{-}\left(\gamma_{k}\right) d\left(\gamma_{k}\right)\right)\left(\gamma_{k}-\zeta\right) \prod_{k^{\prime} \neq k}\left(\gamma_{k}-\gamma_{k^{\prime}}\right)} \\
K & =-\frac{1}{p_{U} L_{+}(\zeta)} \sum_{k=1}^{m} r_{k}\left(1+\left(1-p_{U}\right) d\left(\gamma_{k}\right) L_{-}\left(\gamma_{k}\right)\right) \\
& =\frac{1}{L_{+}(\zeta)} \sum_{k=1}^{m} \frac{P_{+}\left(\gamma_{k}\right)}{\left(\gamma_{k}-\zeta\right) \prod_{k^{\prime} \neq k}\left(\gamma_{k}-\gamma_{k^{\prime}}\right)},
\end{aligned}
$$

it holds for all $x \geq 0$ that

$$
\sum_{k=1}^{m} r_{k} \mathrm{e}^{\gamma_{k} x}+\sum_{k=1}^{m} r_{k} d\left(\gamma_{k}\right) \int_{(-\infty, x]} F(\mathrm{~d} u) \mathrm{e}^{\gamma_{k}(x-u)}+K \int_{(x, \infty)} F(\mathrm{~d} u) \mathrm{e}^{\zeta(x-u)}=0 .
$$

Proof. That (3.15) is the same as (3.16) and (3.18) is the same as (3.17) is immediate from (3.14). (Formally, (3.16) is always well defined because $p_{U}>0,\left|L_{-}\left(\gamma_{k}\right)\right| \leq 1$, and $\left|d\left(\gamma_{k}\right)\right| \leq 1$. Expression (3.15) makes sense only if $d\left(\gamma_{k}\right) \neq 0$, although if $d\left(\gamma_{k}\right)=0$, $R_{+}\left(\gamma_{k}\right)=0$ also by (3.14).) Comparing (3.14) with the Cramér-Lundberg [6, Equation (30)], it is seen that they are the same provided we take

$$
d(\gamma)=\boldsymbol{a}^{\top} \boldsymbol{Q}^{-1}(\gamma, \theta) \lambda,
$$

where the right-hand side comes from [6]. But, then (3.15) and (3.17) match Equations (35) and (42) of [6], and since (3.19) is the same as Equation (43) of [6], the lemma now follows by copying the reasoning given in [6, pp. 977-978], since this reasoning does not depend on the special form $d(\gamma)$ has in [6]. 
Proof of Theorem 3.1. We use the shorthand notation $r_{s}$ for $r\left(Y_{S}^{\circ}\right)$ with $r$ an arbitrary measurable function $r:(E, \mathcal{E}) \rightarrow(\mathbb{R}, \mathcal{B})$.

(i) Because $\operatorname{Re} \gamma<0$ and $\theta>0$, we have

$$
\begin{aligned}
\left|c_{\gamma, \theta}(y)\right| & \leq \mathrm{E}_{y} \int_{0}^{\infty} \lambda_{s} \exp \left(-\int_{0}^{s}\left(\lambda_{u}+\theta\right) \mathrm{d} u\right) \mathrm{d} s \\
& <\mathrm{E}_{y} \int_{0}^{\infty}\left(\lambda_{s}+\theta\right) \exp \left(-\int_{0}^{s}\left(\lambda_{u}+\theta\right) \mathrm{d} u\right) \mathrm{d} s \\
& =1 .
\end{aligned}
$$

That $c_{\gamma, \theta} \in \mathscr{D}_{Y^{\circ}}$ and satisfies (3.5) is essentially a Feynman-Kac-type formula. By (2.9) we need to show that the process (with $c_{\gamma, \theta, t}=c_{\gamma, \theta}\left(Y_{t}^{\circ}\right)$ )

$$
M_{t}=c_{\gamma, \theta, t}-c_{\gamma, \theta}(y)-\int_{0}^{t}\left(\lambda_{s}-\left(\gamma \beta_{s}-\lambda_{s}-\theta\right) c_{\gamma, \theta, s}\right) \mathrm{d} s
$$

is, for every $y \in E$, a mean-zero $\mathrm{P}_{y}$-martingale (with respect to the filtration $\left(\mathcal{F}_{t}^{Y^{\circ}}\right)_{t \geq 0}$ generated by $\left.Y^{\circ}\right)$. The only problem is the martingale property where we show directly that, for $0 \leq t<T$,

$$
\mathrm{E}_{y}\left[M_{T} \mid \widetilde{F}_{t}^{Y^{\circ}}\right]=M_{t}
$$

Define

$$
\alpha(y)=\gamma \beta(y)-\lambda(y)-\theta
$$

so that, for any $y^{\prime} \in E$,

$$
c_{\gamma, \theta}\left(y^{\prime}\right)=-\mathrm{E}_{y^{\prime}} \int_{0}^{\infty} \lambda_{s} \exp \left(\int_{0}^{s} \alpha_{u} \mathrm{~d} u\right) \mathrm{d} s .
$$

By the Markov property,

$$
\mathrm{E}_{y}\left[c_{\gamma, \theta, T} \mid \mathcal{F}_{t}^{Y^{\circ}}\right]=\mathrm{E}_{y^{\prime}}\left[c_{\gamma, \theta, T-t}\right]
$$

where $y^{\prime}$ is the value of $Y_{t}^{\circ}$ specified by the conditioning. But then, again using the Markov property,

$$
\begin{aligned}
\mathrm{E}_{y}\left[c_{\gamma, \theta, T} \mid \mathcal{F}_{t}^{\left.Y^{\circ}\right]}\right. & =-\mathrm{E}_{y^{\prime}} \mathrm{E}_{Y_{T-t}^{\circ}} \int_{0}^{\infty} \lambda_{s} \exp \left(\int_{0}^{s} \alpha_{u} \mathrm{~d} u\right) \mathrm{d} s \\
& =-\mathrm{E}_{y^{\prime}} \int_{0}^{\infty} \lambda_{s+T-t} \exp \left(\int_{0}^{s} \alpha_{u+T-t} \mathrm{~d} u\right) \mathrm{d} s \\
& =-\mathrm{E}_{y^{\prime}} \int_{T-t}^{\infty} \lambda_{s} \exp \left(\int_{T-t}^{s} \alpha_{u} \mathrm{~d} u\right) \mathrm{d} s .
\end{aligned}
$$

Similar calculations give

$$
\mathrm{E}_{y}\left[\int_{0}^{T}\left(\lambda_{s}-\alpha_{s} c_{\gamma, \theta, s}\right) \mathrm{d} s \mid \mathcal{F}_{t}^{Y^{\circ}}\right]=\mathrm{E}_{y^{\prime}} \int_{t}^{T}\left(\lambda_{s-t}+\alpha_{s-t} \int_{s-t}^{\infty} \lambda_{v} \exp \left(\int_{s-t}^{v} \alpha_{u} \mathrm{~d} u\right) \mathrm{d} v\right) \mathrm{d} s,
$$

SO

$$
\mathrm{E}_{y}\left[M_{T} \mid \mathcal{F}_{t}^{Y^{\circ}}\right]=-c_{\gamma, \theta}(y)-\int_{0}^{t}\left(\lambda_{s}-\alpha_{s} c_{\gamma, \theta, s}\right) \mathrm{d} s+\mathrm{E}_{y^{\prime}} I_{t, T}
$$


with

$$
I_{t, T}=-\int_{T-t}^{\infty} \lambda_{s} \exp \left(\int_{T-t}^{s} \alpha_{u} \mathrm{~d} u\right) \mathrm{d} s-\int_{t}^{T}\left(\lambda_{s-t}+\alpha_{s-t} \int_{s-t}^{\infty} \lambda_{v} \exp \left(\int_{s-t}^{v} \alpha_{u} \mathrm{~d} u\right) \mathrm{d} v\right) \mathrm{d} s
$$

Differentiating with respect to $T$ (or using partial integration) shows that $I_{t, T}$ does not depend on $T \geq t$; hence,

$$
I_{t, T}=I_{t, t}=-\int_{0}^{\infty} \lambda_{s} \exp \left(\int_{0}^{s} \alpha_{u} \mathrm{~d} u\right) \mathrm{d} s,
$$

so $\mathrm{E}_{y^{\prime}} I_{t, T}=c_{\gamma, \theta}\left(y^{\prime}\right)=c_{\gamma, \theta, t}$. Referring to (3.20), we thus recognise the right-hand side of (3.21) as $M_{t}$.

That there is only the one bounded solution to (3.5) given by (3.3) is argued as follows. Let $\theta>0$ and $\operatorname{Re} \gamma<0$, and suppose that $c_{\gamma, \theta} \in \mathscr{D}_{Y \circ}$ is a bounded solution to (3.5). Then

$$
\mathrm{d} c_{\gamma, \theta, t}=\mathcal{A}_{Y}{ }^{\circ} c_{\gamma, \theta, t} \mathrm{~d} t+\mathrm{d} M_{t}
$$

with $M$ a mean-zero martingale. But, also defining

$$
Z_{t}:=c_{\gamma, \theta, t} \exp \left(\int_{0}^{t} \alpha_{s} \mathrm{~d} s\right)
$$

we then have, because of (3.5),

$$
\mathrm{d} Z_{t}=\left(\alpha_{t} c_{\gamma, \theta, t}+\mathcal{A}_{Y^{\circ}} c_{\gamma, \theta, t}\right) \exp \left(\int_{0}^{t} \alpha_{s} \mathrm{~d} s\right) \mathrm{d} t+\mathrm{d} M_{t}^{*}=\lambda_{t} \exp \left(\int_{0}^{t} \alpha_{s} \mathrm{~d} s\right) \mathrm{d} t+\mathrm{d} M_{t}^{*},
$$

where

$$
\mathrm{d} M_{t}^{*}=\exp \left(\int_{0}^{t} \alpha_{s} \mathrm{~d} s\right) \mathrm{d} M_{t}
$$

is a new mean-zero martingale (a true martingale since $\left|\exp \int_{0}^{t} \alpha_{s} \mathrm{~d} s\right| \leq 1$; see Remark 2.1). For any $y \in E$, we therefore have

$$
\mathrm{E}_{y} Z_{t}=c_{\gamma, \theta}(y)+\mathrm{E}_{y} \int_{0}^{t} \lambda_{s} \exp \left(\int_{0}^{s} \alpha_{u} \mathrm{~d} u\right) \mathrm{d} s .
$$

As $t \rightarrow \infty, \operatorname{Re} \int_{0}^{t} \alpha_{s} \mathrm{~d} s \rightarrow-\infty$ (because $\operatorname{Re} \alpha_{s} \leq-\theta<0$ ), so $c_{\gamma, \theta}(y)$ is bounded, and dominated convergence when taking expectations in (3.22) yields $\lim _{t \rightarrow \infty} \mathrm{E}_{y} Z_{t}=0$ and (3.3) follows.

(ii) As in [5] and [6], the idea is to look for a bounded partial eigenfunction for the generator $\mathcal{A}_{X, Y}$ of $(X, Y)$. More precisely, for each $\theta>0$, we shall find a $\mathbb{C}$-valued function $h$ such that

$$
\mathcal{A}_{X, Y} h(x, y)=\theta h(x, y), \quad x \geq 0, y \in E .
$$

Inspired by [6] and assuming that (3.6) has $m$ distinct solutions $\gamma_{1}(\theta), \ldots, \gamma_{m}(\theta)$, denoted by $\gamma_{k}$ in the sequel, it is natural to look for $h$ of the form

$$
h(x, y)= \begin{cases}\sum_{k=1}^{m} r_{k} c_{\gamma_{k}, \theta}(y) \mathrm{e}^{\gamma_{k} x}, & x \geq 0, y \in E, \\ K \mathrm{e}^{\zeta x}, & x<0, y \in E,\end{cases}
$$

where $\zeta \geq 0$ and $c_{\gamma_{k}, \theta}$ is the bounded solution to (3.5) obtained for $\gamma=\gamma_{k}$. If this $h$ satisfies (3.24), Lemma 3.1 immediately gives

$$
K \mathbb{E}_{x_{0}, y_{0}} \mathrm{e}^{-\theta \tau-\zeta Z}=h\left(x_{0}, y_{0}\right), \quad x_{0} \geq 0, y_{0} \in E ;
$$


hence, (3.7) follows if we show that (3.24) holds with the $r_{k}$ given by (3.9) and $K$ given by (3.18). By straightforward computations, for $x \geq 0$ and $y \in E$, using (2.10),

$$
\begin{aligned}
\left(\mathcal{A}_{X, Y}-\theta I\right) h(x, y)= & \sum_{k=1}^{m} r_{k} \mathrm{e}^{\gamma_{k} x}\left[\left(\gamma_{k} \beta(y)-\lambda(y)-\theta\right) c_{\gamma_{k}, \theta}(y)+\mathcal{A}_{Y^{\circ}} c_{\gamma_{k}, \theta}(y)\right] \\
& +\lambda(y) \sum_{k=1}^{m} r_{k}\left(\int_{E} A(\mathrm{~d} v) c_{\gamma_{k}, \theta}(v)\right) \int_{(-\infty, x]} F(\mathrm{~d} u) \mathrm{e}^{\gamma_{k}(x-u)} \\
& +\lambda(y) K \int_{(x, \infty)} F(\mathrm{~d} u) \mathrm{e}^{\zeta(x-u)}
\end{aligned}
$$

The key observation and the raison d'être for (3.5) is that, because of (3.5), the sum in the first line reduces to $\lambda(y) \sum_{k=1}^{m} r_{k} \mathrm{e}^{\gamma_{k} x}$, and, with the $r_{k}$ given by (3.9), and $K$ given by (3.17), it follows from Lemma 3.2 with

$$
d(\gamma)=\int_{E} A(\mathrm{~d} v) c_{\gamma, \theta}(v)
$$

that $h$ given by (3.25) is the desired partial eigenfunction since, by (3.4), $|d(\gamma)|<1$.

It remains to show that (3.6) has exactly $m$ solutions $\gamma$ with $\operatorname{Re} \gamma<0$. But, it is fairly easy to argue that (3.4) implies this: by assumption, $L_{-}(\gamma)$ and $d(\gamma)$ are analytic functions of $\gamma \in D(\theta)$. Hence, by Rouché's theorem from complex function theory we need only show that, for sufficiently large $S>0$, it holds for $z \in \partial \Gamma_{S}$ that

$$
\left|R_{+}(z)\left(1-p_{U}\right) L_{-}(z) d(z)+p_{U} P_{+}(z) d(z)\right|<\left|R_{+}(z)\right|,
$$

where $\partial \Gamma_{S}$ denotes the boundary of the region

$$
\Gamma_{S}=\{z \in \mathbb{C}: \operatorname{Re} z<0,|z| \leq S\} \cup\{z \in \mathbb{C}: z=\mathrm{i} w,-S \leq w \leq S\} .
$$

(Inequality (3.27) implies that the number of solutions to (3.6) with $\gamma$ inside $\Gamma_{S}$ is the same as the number of roots for $R_{+}$inside $\Gamma_{S}$ and this number is exactly $m$, the degree of $R_{+}$, if $S$ is sufficiently large. Also, $\bigcup_{S>0} \Gamma_{S}=\{z \in \mathbb{C}: \operatorname{Re} z \leq 0\}$.)

Since $P_{+}$is a polynomial of degree less than $m$, we have $\left|P_{+}(z)\right|<\left|R_{+}(z)\right|$ for sufficiently large $|z|$. Furthermore, by (3.1), $\left|P_{+}(\mathrm{i} w)\right| \leq\left|R_{+}(\mathrm{i} w)\right|$ for $w \in \mathbb{R}$; hence, $\left|P_{+}(z)\right| \leq\left|R_{+}(z)\right|$ for $z \in \partial \Gamma_{S}$ if $S$ is sufficiently large. Because $p_{U}>0$ and $\left|L_{-}(z)\right| \leq 1$ if $\operatorname{Re} z \leq 0$, (3.27) now follows using (3.4).

Remark 3.3. As mentioned at the end of the introduction, it may be possible to solve the ruin problem for the Albrecher-Boxma model [1] using partial eigenfunctions. The risk process in the model has the standard form

$$
X_{t}=x_{0}+c t-\sum_{n=1}^{N_{t}} U_{n}
$$

with $c>0$ and $U_{n}>0$. The occurrence and size of the jumps are determined from a Markov chain $J^{\mathrm{d}}=\left(J_{n}^{\mathrm{d}}\right)_{n \geq 0}$ in discrete time with finite state space $H=\{1, \ldots, M\}$ and transition probabilities $\left(p_{i j}\right)_{i, j \in H}$ together with a collection of given jump intensities $\left(\lambda_{i}\right)_{i \in H}$ and a given family $\left(F_{j}\right)_{j \in H}$ of distributions on $(0, \infty)$ in the following manner: with $J_{0}^{\mathrm{d}} \equiv i_{0} \in H$, let 
$\tau_{1}$ be exponential at rate $\lambda_{i_{0}}$ and, given $\tau_{1}$ and $J_{1}^{\mathrm{d}}=i_{1}$, let $U_{1}$ have distribution $F_{i_{1}}$; given $\tau_{1}$, $J_{1}^{\mathrm{d}}=i_{1}$, and $U_{1}$, let $T^{(2)}=\tau_{2}-\tau_{1}$ be exponential at rate $\lambda_{i_{1}}$ and, given in addition $J_{2}^{\mathrm{d}}=i_{2}$, let $U_{2}$ have distribution $F_{i_{2}}$; continue in the obvious manner.

Defining $J_{t}=J_{N_{t}}^{\mathrm{d}}$, the joint process $(X, J)$ is a time-homogeneous Markov process with generator of the form

$$
\mathcal{A}_{X, J} f(x, i)=c \frac{\partial}{\partial x} f(x, i)+\lambda_{i} \sum_{j \in H} p_{i j} \int_{(0, \infty)} F_{j}(\mathrm{~d} s)(f(x-s, j)-f(x, i)) .
$$

In [1] the authors were interested in determining the discounted penalty function at ruin (GerberShiu-type functional), which in our notation is the expectation

$$
\mathbb{E}_{x, i}\left[\mathrm{e}^{-\theta \tau} w\left(X_{\tau-}, Z\right) ; \tau<\infty\right]
$$

for $x \geq 0$. The results discussed above correspond to the simple choice $w(x, z)=\mathrm{e}^{-\zeta z}$, nevertheless it is of interest to ask whether, for this choice of $w$, a result similar to Theorem 3.1 can be obtained using partial eigenfunctions since they may well be more explicit in form than those given [1]. Comparing generator (2.10) with (3.28), it is seen that the overlap corresponds to (writing $a_{j}$ for $A(\{j\})$ )

$$
\mathcal{A}_{X, J} f(x, i)=c \frac{\partial}{\partial x} f(x, i)+\lambda_{i} \sum_{j \in H} a_{j} \int_{(0, \infty)} F(\mathrm{~d} s)(f(x-s, j)-f(x, i)),
$$

i.e. the special case of the Albrecher-Boxma model treated in this paper corresponds to taking $Y^{\circ}$ completely deterministic (and not equivalent to $J$ ) with $Y_{t}^{\circ}=Y_{0}^{\circ}$ for all $t, \beta(i)=c$, $p_{i j}=a_{j}$, and $F_{j} \equiv F$, which is a very simple model.

In order to use partial eigenfunctions on the Albrecher-Boxma model, it is necessary to at least require that each $F_{j}$ has a rational Laplace transform. One may then try to look for a partial eigenfunction $h$ satisfying, for some finite index set $K$,

$$
h(x, i)=\sum_{k \in K} d_{i k} \mathrm{e}^{\gamma_{k} x}
$$

for $x \geq 0$ and all $i \in H$ with all $\operatorname{Re} \gamma_{k} \leq 0$; cf. (3.25). The problems that arise consist in determining the size of $K$ (in many cases there is a natural guess) and how to find the $d_{i k}$ and the $\gamma_{k}$. Finding an equation for the $\gamma_{k}$ appears particularly challenging, it is not at all clear which equation to use instead of the Cramér-Lundberg equation (3.6)! We do believe however that, by restricting all $F_{j}$ to be distributions that are finite linear combinations of exponentials, it is indeed possible to find partial eigenfunctions of the form (3.29), but it does appear essential that $Y^{\circ}$ is as simple as a finite state space Markov chain. These results will hopefully appear in future work.

We shall now discuss how to find the marginal Laplace transform (2.12). For $\gamma$ with $\operatorname{Re} \gamma \leq 0$, define, in analogy with (3.3), taking $\theta=0$ there,

$$
c_{\gamma, 0}(y)=-\mathrm{E}_{y} \int_{0}^{\infty} \lambda\left(Y_{s}^{\circ}\right) \exp \left(\int_{0}^{s}\left(\gamma \beta\left(Y_{u}^{\circ}\right)-\lambda\left(Y_{u}^{\circ}\right)\right) \mathrm{d} u\right) \mathrm{d} s .
$$

Note that $\left|c_{\gamma, 0}(y)\right| \leq 1$. The case $\gamma=0$ is particularly important:

$$
c_{0,0}(y)=-1+\mathrm{E}_{y} \exp \left(-\int_{0}^{\infty} \lambda\left(Y_{s}^{\circ}\right) \mathrm{d} s\right) .
$$


For the statement of the final result, we retain the assumptions from Theorem 3.1 concerning $L_{+}, R_{+}$, and $L_{-}$, and the functions $c_{\gamma, \theta}$ when $\theta>0$ and $\operatorname{Re} \gamma<0$.

The reader is reminded of the concepts of $A$-jumping, $A$-recurrence, and $A$-transience introduced at the end of Section 2.

Theorem 3.2. (i) Suppose that, for every $\gamma \in \mathbb{C}$ with $\operatorname{Re} \gamma<0$, the function $c_{\gamma, 0}$ defined by (3.30) is continuous. Then $c_{\gamma, 0}$ is a bounded solution to the functional equation

$$
(\gamma \beta(y)-\lambda(y)) c_{\gamma, 0}(y)+\mathcal{A}_{Y^{\circ}} c_{\gamma, 0}(y)=\lambda(y), \quad y \in E .
$$

Any bounded and continuous solution to this equation is uniquely determined at all $y \in E$ for which (2.7) holds, i.e.

$$
\mathrm{P}_{y}\left(\int_{0}^{\infty} \lambda\left(Y_{s}^{\circ}\right) \mathrm{d} s=\infty\right)=1 .
$$

(ii) Still assuming that the $c_{\gamma, 0}$ are continuous, the modified Cramér-Lundberg equation

$$
R_{+}(\gamma)\left(1+\left(1-p_{U}\right) L_{-}(\gamma) \int_{E} A(\mathrm{~d} v) c_{\gamma, 0}(v)\right)=-p_{U} P_{+}(\gamma) \int_{E} A(\mathrm{~d} v) c_{\gamma, 0}(v)
$$

has precisely $m$ solutions $\gamma_{1}(0), \ldots, \gamma_{m}(0)$ (counted with multiplicity) that satisfy the following conditions:

- if $(X, Y)$ is A-transient, $\operatorname{Re} \gamma_{k}(0)<0$ for $1 \leq k \leq m$;

- if $(X, Y)$ is A-recurrent, one of the solutions, $\gamma_{m}(0)$ say, equals 0 and $\operatorname{Re} \gamma_{k}(0)<0$ for $1 \leq k \leq m-1$;

- if $(X, Y)$ is not A-jumping, $\operatorname{Re} \gamma_{k}(0)<0$ for $1 \leq k \leq m$.

If these $m$ solutions are distinct then, for all $\zeta>0$,

$$
\mathbb{E}_{x, y}\left[\mathrm{e}^{-\zeta Z} ; \tau<\infty\right]=\frac{L_{+}(\zeta) \sum_{k=1}^{m} r_{k} c_{\gamma_{k}(0), 0}(y) \mathrm{e}^{\gamma_{k}(0) x}}{\sum_{k=1}^{m} q_{k}}, \quad x \geq 0, y \in E,
$$

with

$$
\begin{aligned}
q_{k} & =\frac{P_{+}\left(\gamma_{k}(0)\right)}{\left(\gamma_{k}(0)-\zeta\right) \prod_{k^{\prime} \neq k}\left(\gamma_{k}(0)-\gamma_{k^{\prime}}(0)\right)} \\
r_{k} & =-\frac{p_{U} q_{k}}{1+\left(1-p_{U}\right) L_{-}\left(\gamma_{k}\right) \int_{E} A(\mathrm{~d} v) c_{\gamma_{k}(0), 0}(v)}
\end{aligned}
$$

For $\zeta=0$, corresponding to finding the ruin probability $\mathbb{P}_{x, y}(\tau<\infty)$, (3.35) still holds if $(X, Y)$ is either A-transient or not A-jumping. If $(X, Y)$ is A-recurrent then

$$
\mathbb{P}_{x, y}(\tau<\infty)=1-\mathrm{E}_{y} \exp \left(-\int_{0}^{\infty} \lambda\left(Y_{s}^{\circ}\right) \mathrm{d} s\right), \quad x \geq 0, y \in E .
$$

Remark 3.4. Note that the ruin probability (3.38) in the $A$-recurrent case is precisely $\mathbb{P}_{y}\left(\tau_{1}<\infty\right)$, as it should be; cf. the discussion at the end of Section 2 .

Remark 3.5. For $\gamma=0$, (3.32) always has the solution $c_{0,0} \equiv-1$, and, referring to (3.31), we see that if, for some $y, \mathrm{P}_{y}\left(\int_{0}^{\infty} \lambda\left(Y_{s}^{\circ}\right) \mathrm{d} s<\infty\right)>0$ then (3.32) for $\gamma=0$ has at least two linearly independent bounded and continuous solutions. 
Proof of Theorem 3.2. (i) Assume that, with $\operatorname{Re} \gamma \leq 0, c_{\gamma, 0}$ given by (3.30) is continuous. A word-for-word copy of the proof that $\left(M_{t}\right)$ defined by (3.20) is a martingale shows that

$$
M_{t}=c_{\gamma, 0, t}-c_{\gamma, 0}(y)-\int_{0}^{t}\left(\lambda_{s}-\left(\gamma \beta_{s}-\lambda_{s}\right) c_{\gamma, 0, s}\right) \mathrm{d} s
$$

is a martingale, so $c_{\gamma, 0} \in \mathscr{D}_{Y}$ and satisfies (3.32).

Suppose conversely that $\tilde{c}_{\gamma, 0}$ is a bounded and continuous solution to (3.32). Then, defining (see (3.22))

$$
\tilde{Z}_{t}:=\tilde{c}_{\gamma, 0, t} \exp \left(\int_{0}^{t}\left(\gamma \beta_{s}-\lambda_{s}\right) \mathrm{d} s\right)
$$

we find, exactly as in the proof of (3.23), that

$$
\mathrm{E}_{y} \tilde{Z}_{t}=\tilde{c}_{\gamma, 0}(y)+\mathrm{E}_{y} \int_{0}^{t} \lambda_{s} \exp \left(\int_{0}^{s}\left(\gamma \beta_{u}-\lambda_{u}\right) \mathrm{d} u\right) \mathrm{d} s,
$$

whence

$$
\lim _{t \rightarrow \infty} \mathrm{E}_{y} \tilde{Z}_{t}=\tilde{c}_{\gamma, 0}(y)-c_{\gamma, 0}(y)
$$

so uniqueness at $y$ holds precisely when the limit on the left-hand side is 0 . But, if (3.33) holds, this is true since then $\lim _{t \rightarrow \infty} \tilde{Z}_{t}=0, \mathrm{P}_{y}$-a.s.

(ii) We shall use the fact that

$$
\mathbb{E}_{x, y}\left[\mathrm{e}^{-\zeta Z} ; \tau<\infty\right]=\lim _{\theta \rightarrow 0, \theta>0} \mathbb{E}_{x, y} \mathrm{e}^{-\theta \tau-\zeta Z}
$$

Consider the solutions $\gamma_{1}(\theta), \ldots, \gamma_{m}(\theta)$ to (3.6) for an arbitrary $\theta>0$ with all $\operatorname{Re} \gamma_{k}(\theta)<0$ in some order (e.g. increasing real part first, increasing imaginary part second). Pick an arbitrary sequence $\theta_{n}>0$ with $\theta_{n} \rightarrow 0$. We claim that there is a subsequence $n^{\prime}$ such that the limit

$$
\left(\gamma_{1}(0), \ldots, \gamma_{m}(0)\right)=\lim _{n^{\prime} \rightarrow \infty}\left(\gamma_{1}\left(\theta_{n^{\prime}}\right), \ldots, \gamma_{m}\left(\theta_{n^{\prime}}\right)\right)
$$

exists. To see this, find $S_{0}>0$ such that $\left|P_{+}(\gamma)\right|<\frac{1}{2}\left|R_{+}(\gamma)\right|$ for all $\gamma \in \mathbb{C}$ with $|\gamma|>S_{0}$. Rewriting (3.6) as

$$
R_{+}(\gamma)=\left(-p_{U} P_{+}(\gamma)-\left(1-p_{U}\right) R_{+}(\gamma) L_{-}(\gamma)\right) \int_{E} A(\mathrm{~d} v) c_{\gamma, \theta}(v),
$$

it is immediately seen that, for $|\gamma|>S_{0}$, the expression on the right-hand side is in absolute value less than or equal to

$$
p_{U}\left|P_{+}(\gamma)\right|+\left(1-p_{U}\right)\left|R_{+}(\gamma)\right|<\left(\frac{1}{2} p_{U}+\left(1-p_{U}\right)\right)\left|R_{+}(\gamma)\right|<\left|R_{+}(\gamma)\right|,
$$

and, consequently, any solution $\gamma(\theta)$ to (3.6) satisfies $|\gamma(\theta)| \leq S_{0}$. In particular, all $\left|\gamma_{k}\left(\theta_{n}\right)\right| \leq$ $S_{0}$ and (3.39) follows by compactness. Obviously, $\operatorname{Re} \gamma_{k}(0) \leq 0$ for all $k$.

Next, observe that, by dominated convergence, $\lim _{n^{\prime}} c_{\gamma_{k}\left(\theta_{n^{\prime}}\right), \theta_{n^{\prime}}}(y)=c_{\gamma_{k}(0), 0}(y)$ for all $y$; cf. (3.3) and (3.30). Using (3.7) with $\theta=\theta_{n^{\prime}}$ and letting $n^{\prime \prime} \rightarrow \infty$, for $\zeta>0$ (!), we now obtain (3.35) provided the $\gamma_{k}(0)$ are distinct and with the relevant $q_{k}$ and $r_{k}$ in (3.36) and (3.37) obtained by taking limits in (3.8) and (3.9).

Note that, because of (3.35), we can conclude that the limit point $\left(\gamma_{1}(0), \ldots, \gamma_{m}(0)\right)$ is uniquely determined (up to permutations of the entries) since if not, different functions of $(x, y)$ yielding the same expectation would appear. 
Taking limits in (3.6) shows that all of the $\gamma_{k}(0)$ are solutions to (3.34), an equation which therefore has at least $m$ solutions $\gamma$ (counted with multiplicity) with $\operatorname{Re} \gamma \leq 0$. We see directly that $\gamma=0$ is a solution to (3.34) if and only if

$$
\int_{E} A(\mathrm{~d} v) c_{0,0}(v)=-1
$$

i.e. if and only if $c_{0,0}(y)=-1, A$-a.s.

It was assumed above that $\zeta>0$. For $\zeta=0$, we have $L_{+}(\zeta)=1$ and (3.35) is still valid provided all $\gamma_{k}(0) \neq 0$, in which case (3.35) the yields the ruin probability $\mathbb{P}_{x, y}(\tau<\infty)$. If, however, $\zeta=0$ and one of the $\gamma_{k}(0)$, e.g. $\gamma_{m}(0)$ equals 0 , because of the appearance of the factor $\gamma_{m}(0)$ in the denominator, $q_{m}$ is not defined by (3.36). In this case, before taking limits on the right-hand side of (3.7), multiply both the numerator and denominator by $\gamma_{m}\left(\theta_{n^{\prime}}\right)$ and then obtain the desired limit

$$
\mathbb{P}_{x, y}(\tau<\infty)=-\frac{p_{U}}{1+\left(1-p_{U}\right) \int_{E} A(\mathrm{~d} v) c_{0,0}(v)} c_{0,0}(y)=-c_{0,0}(y),
$$

where the last equality holds because of (3.40).

It remains to show that if $\gamma_{k}(0) \neq 0$ then $\operatorname{Re} \gamma_{k}(0)<0$ and to argue that (3.34) has precisely the solutions claimed in statement (ii).

The first claim is easy: we know that $\operatorname{Re} \gamma_{k}(0) \leq 0$, so assume that $\gamma_{k}(0)=\mathrm{i} w$ for some $w \in \mathbb{R} \backslash 0$. From (3.34), it follows that

$$
\int_{E} A(\mathrm{~d} v) c_{\mathrm{i} w, 0}(v)=\frac{-1}{p_{U} L_{+}(\mathrm{i} w)+\left(1-p_{U}\right) L_{-}(\mathrm{i} w)},
$$

and, since $w \neq 0, p_{U}>0$, and $F_{+}$is not degenerate at 0 , the absolute value of the denominator on the right-hand side is less than 1 . Since $\left|c_{\mathrm{i} w, 0}\right| \leq 1$, we have reached a contradiction.

The second claim is more difficult. Suppose that all $\operatorname{Re} \gamma_{k}(0)<0$. Then (3.35) with $\zeta=0$ gives $\lim _{x \rightarrow \infty} \mathbb{P}_{x, y}(\tau<\infty)=0$ and (2.13) implies that $(X, Y)$ is not $A$-recurrent, i.e. either $A$-transient or not $A$-jumping. If one of the $\gamma_{k}(0)$ equals 0 , using (3.41) and (2.13) we see that, for $x \geq 0$ and $y \in E$,

$$
\mathbb{P}_{x, y}(\tau<\infty)=-c_{0,0}(y)=\mathbb{P}_{y}\left(\tau_{1}<\infty\right),
$$

which certainly fits with $(X, Y)$ being $A$-recurrent. Since (2.14) also holds if $(X, Y)$ is not $A$-recurrent, we conclude that all $\operatorname{Re} \gamma_{k}(0)<0$ in the non- $A$-recurrent case and that in the $A$-recurrent case $m-1$ of the $\gamma_{k}(0)$ have strictly negative real parts with the remaining $\gamma_{k}(0)$ equal to 0 .

We finally need to argue that (3.34) has no solutions $\gamma$ with $\operatorname{Re} \gamma<0$ other than the $\gamma_{k}(0)$ found for the $A$-transient, $A$-recurrent, and non- $A$-jumping cases. The latter is simple since the argument involving Rouché's theorem in the proof of Theorem 3.1 applies unchanged. To deal with the $A$-jumping case, assume that (3.34), apart from the $\gamma_{k}(0)$, has an additional solution $\gamma_{m+1} \in \mathbb{C}_{-}$, different from all the $\gamma_{k}(0)$ with, necessarily, $\operatorname{Re} \gamma_{m+1}<0$. Assuming that the solution 0 to (3.34) in the $A$-recurrent case is $\gamma_{m}(0)$, define

$$
\left(\tilde{\gamma}_{1}, \ldots, \tilde{\gamma}_{m}\right)=\left(\gamma_{1}(0), \ldots, \gamma_{m-1}(0), \gamma_{m+1}\right)
$$

Using Lemma 3.2 with $d(\gamma)=\int_{E} A(\mathrm{~d} v) c_{\gamma, 0}(v)$, it follows that (3.19) holds with $r_{k}=\tilde{r}_{k}$ and $K=\tilde{K}$, where $\tilde{r}_{k}$ and $\tilde{K}$ are given by (3.16) and (3.18), respectively, when taking $\gamma_{k}=\tilde{\gamma}_{k}$. 
Defining, cf. (3.25),

$$
\tilde{h}(x, y)= \begin{cases}\sum_{k=1}^{m} \tilde{r}_{k} c_{\widetilde{\gamma}_{k}, \theta}(y) \mathrm{e}^{\tilde{\gamma}_{k} x}, & x \geq 0, y \in E, \\ \tilde{K} \mathrm{e}^{\zeta x}, & x<0, y \in E,\end{cases}
$$

using (3.19) and (3.32), we find that $\mathcal{A}_{X, Y} \tilde{h}(x, y)=0$ for $x \geq 0$ and $y \in E$, so, by (3.13), from Lemma 3.1,

$$
\mathbb{E}_{x, y} \tilde{h}\left(X_{\tau \wedge t}, Y_{\tau \wedge t}\right)=\tilde{h}(x, y) .
$$

Letting $t \rightarrow \infty$ gives

$$
\tilde{h}(x, y)=\mathbb{E}_{x, y}\left[\tilde{K} \mathrm{e}^{\zeta X_{\tau}} ; \tau<\infty\right]+\lim _{t \rightarrow \infty} \mathbb{E}_{x, y}\left[\tilde{h}\left(X_{t}, Y_{t}\right) ; \tau=\infty\right] .
$$

In the $A$-transient case we have $X_{t} \rightarrow \infty$ on $\left(\tau_{1}<\infty\right)$ and, therefore, by (3.42), $\tilde{h}\left(X_{t}, Y_{t}\right) \rightarrow 0$, $\mathbb{P}_{x, y}$-a.s. on $\left(\tau_{1}<\infty\right)$. In the $A$-recurrent case, on $(\tau=\infty)$ we have $\tau_{1}=\infty, \mathbb{P}_{x, y}$-a.s. Consequently, in both of these cases

$$
\begin{aligned}
\lim _{t \rightarrow \infty} & \mathbb{E}_{x, y}\left[\tilde{h}\left(X_{t}, Y_{t}\right) ; \tau=\infty\right] \\
& =\lim _{t \rightarrow \infty} \mathbb{E}_{x, y}\left[\tilde{h}\left(X_{t}, Y_{t}\right) ; \tau_{1}=\infty\right] \\
& =\lim _{t \rightarrow \infty} \mathrm{E}_{y} \exp \left(-\int_{0}^{\infty} \lambda\left(Y_{s}^{\circ}\right) \mathrm{d} s\right) \sum_{k=1}^{m} \tilde{r}_{k} c_{\gamma_{k}, 0}\left(Y_{t}^{\circ}\right) \exp \left(\tilde{\gamma}_{k}\left(x+\int_{0}^{t} \beta\left(Y_{s}^{\circ}\right) \mathrm{d} s\right)\right) \\
& =0
\end{aligned}
$$

where the last equality is argued below. For the non- $A$-jumping case, a modified argument using the fact that $X$ has only finitely many jumps shows that the limit of the last term in (3.43) is still 0. Thus, in all cases, from (3.43) and using (3.42) for $x \geq 0$, it follows that

$$
\mathbb{E}_{x, y}\left[\mathrm{e}^{-\zeta Z} ; \tau<\infty\right]=\frac{1}{\widetilde{K}} \sum_{k=1}^{m} \tilde{r}_{k} \tilde{c}_{\widetilde{\gamma}_{k}, 0} \mathrm{e}^{\tilde{\gamma}_{k} x}
$$

where the presence of the term involving $\mathrm{e}^{\gamma_{m+1} x}$ contradicts the established result (3.35) and we may therefore conclude that an extra solution $\gamma_{m+1}$ to (3.34) with $\operatorname{Re} \gamma_{m+1}<0$ cannot exist.

We conclude by showing that (3.44) holds: ignoring the factor $\tilde{r}_{k}$, in absolute value the $k$ th term is less than or equal to

$$
\mathrm{E}_{y} \exp \left(-\int_{0}^{t} \lambda\left(Y_{s}^{\circ}\right) \mathrm{d} s\right)\left|\tilde{c}_{\widetilde{\gamma}_{k}, 0}\left(Y_{t}^{\circ}\right)\right|
$$

Writing $y^{\prime}=Y_{t}^{\circ}$ we have

$$
\left|\tilde{c}_{\widetilde{\gamma}_{k}, 0}\left(y^{\prime}\right)\right| \leq \mathrm{E}_{y^{\prime}}\left(1-\exp \left(-\int_{0}^{\infty} \lambda\left(Y_{s}^{\circ}\right) \mathrm{d} s\right)\right),
$$

so, by the Markov property, the expression in (3.45) is less than or equal to

$$
\exp \left(-\int_{0}^{t} \lambda\left(Y_{s}^{\circ}\right) \mathrm{d} s\right)\left(1-\exp \left(-\int_{t}^{\infty} \lambda\left(Y_{s}^{\circ}\right) \mathrm{d} s\right)\right) \rightarrow 0 \quad \text { as } t \rightarrow \infty .
$$




\section{Examples}

We shall present three special cases of Theorem 3.1 corresponding to $Y^{\circ}$ a Markov chain on a finite state space (Example 4.1), $Y^{\circ}$ completely deterministic (Example 4.2), and $Y^{\circ}$ a Cox-Ingersoll-Ross diffusion (Example 4.3).

Example 4.1. Suppose that $Y^{\circ}$ is a homogeneous Markov chain on a finite state space $E$ equipped with the $\sigma$-algebra $E$ of all subsets of $E$. The model is the special case of that considered in [6], see (4) therein, corresponding to taking all $\sigma_{i}=0$ and all $\beta_{i} \geq 0$. Denoting elements of $E$ by $i, j$ and writing $g_{i}$ instead of $g(i)$ for function values, we have

$$
\left(\mathcal{A}_{Y} \circ g\right)_{i}=\sum_{j \in E} q_{i j}\left(g_{j}-g_{i}\right),
$$

where $\left(q_{i j}\right)$ is the transition intensity matrix for $Y^{\circ}$. In particular, $q_{i}:=-q_{i i}=\sum_{\{j: j \neq i\}} q_{i j}$ and (3.5) becomes, writing $\boldsymbol{g}$ for the column vector of values $g_{i}$ of a function $g$,

$$
\boldsymbol{Q}(\gamma, \theta) \boldsymbol{c}_{\gamma}=\lambda
$$

where $\boldsymbol{Q}(\gamma, \theta)$ is the matrix with entries

$$
q_{i j}(\gamma, \theta)= \begin{cases}\gamma \beta_{i}-q_{i}-\lambda_{i}-\theta, & \text { if } i=j, \\ q_{i j}, & \text { if } i \neq j\end{cases}
$$

in agreement with Equation (25) of [6]. Also, $\int A(\mathrm{~d} v) c_{\gamma}(v)$ becomes

$$
\boldsymbol{a}^{\top} \boldsymbol{Q}^{-1}(\gamma, \theta) \lambda
$$

hence, the Cramér-Lundberg equation (3.6) is the same as that given in Equation (30) of [6] and it is easily seen that (3.7) is the same as Equation (34) from Theorem 1 of [6] when remembering that in the setup in the present paper, ruin by creeping is impossible.

Example 4.2. One choice of $Y^{\circ}$ that makes it possible to solve (3.5) explicitly is when $Y^{\circ}$ is $\mathbb{R}$-valued and completely deterministic, i.e.

$$
Y_{t}^{\circ}=\phi_{t}\left(Y_{0}^{\circ}\right)
$$

with the $\phi_{t}: E \rightarrow E$ (where $E \subseteq \mathbb{R}$ ) forming a semigroup under composition:

$$
\phi_{0}=\mathrm{id}, \quad \phi_{t} \circ \phi_{s}=\phi_{t+s}, \quad s, t \geq 0 .
$$

Assuming that $t \mapsto \phi_{t}(y)$ is differentiable for all $y$, we have, for $g$ differentiable (or just absolutely continuous),

$$
\mathcal{A}_{Y^{\circ}} g(y)=a(y) g^{\prime}(y)
$$

where $a(y)=\left.D_{t} \phi_{t}(y)\right|_{t=0}$. ( $Y^{\circ}$ is a special and extreme example of a piecewise-deterministic Markov process as introduced in [3]. See also [7, pp. 159-160 and Equation (7.58)] for a discussion of the structure of composition semigroups and the form of the generator.)

A first and very simple particular case of this deterministic model for $Y^{\circ}$ is when $E=(0, \infty)$ and $\beta(y), \lambda(y)$, and $a(y)$ are proportional, e.g. assume that $a(y)>0$ for $y>0$ (forcing 
$t \mapsto \phi_{t}(y)$ to be strictly increasing) with $\beta(y)=\beta a(y)$ and $\lambda(y)=\lambda a(y)$, where $\beta>0$ and $\lambda>0$ are constants. Then (3.32) becomes $(\gamma \beta-\lambda) c_{\gamma, 0}(y)+c_{\gamma, 0}^{\prime}(y)=\lambda$, i.e.

$$
c_{\gamma, 0}(y)=\frac{\lambda}{\gamma \beta-\lambda}+K \exp -(\gamma \beta-\lambda) y .
$$

With $\operatorname{Re} \gamma \leq 0$ and $y>0$, the unique bounded solution is the constant

$$
c_{\gamma, 0}(y)=\frac{\lambda}{\gamma \beta-\lambda},
$$

and it follows that the Cramér-Lundberg equation (3.34) is the same for all choices of the function $a$ and the reset distribution $A$. At first this may seem surprising since Theorem 3.2 now implies that the $\mathbb{P}_{x_{0}, y_{0}}$-probability of ruin depends neither on $a$, $A$, nor $y$. That this is so becomes clear however when one realises that the $\mathbb{P}_{x_{0}, y_{0}}$-distribution of the random variable $X_{T^{(1)}-}-x_{0}$ is the exponential distribution with rate parameter $\lambda / \beta$ for all $a, A$, and $x_{0} \geq 0$, $y_{0}>0$ ! Thus, the ruin probability for $X$ starting from $x_{0}$ is simply the probability that a random walk $x_{0}-\sum_{k=1}^{n} \tilde{U}_{k}$ in discrete time started from $x_{0}$ ever becomes less than 0 with the $\tilde{U}_{k}$ i.i.d., having the same distribution as $U_{k}-V_{k}$ and the $V_{k}$ i.i.d., independent of the $U_{k}$ and exponential at rate $\lambda / \beta$. (That $X_{T^{(1)}-}-x_{0}$ is exponential may be seen as follows. For a given $y_{0}>0$, introduce $\Phi(t)=\int_{0}^{t} \phi_{s}\left(y_{0}\right) \mathrm{d} s$. Then $X_{T^{(1)}-}-x_{0}=\beta \Phi\left(T^{(1)}\right)$ and the $\mathrm{P}_{y_{0}}$-distribution of $T^{(1)}$ has density $f_{T^{(1)}}(t)$ given by

$$
f_{T^{(1)}}(t)=\lambda \phi_{t}\left(y_{0}\right) \mathrm{e}^{-\lambda \Phi(t)} .
$$

Thus, the $\mathrm{P}_{x_{0}, y_{0}}$-distribution of $X_{T_{1}-}-x_{0}$ (which, by the additivity of $X$, does not depend on $\left.x_{0}\right)$ is given by

$$
\mathbb{P}_{y_{0}}\left(X_{T^{(1)}-}-x_{0} \in \mathrm{d} z\right)=\frac{1}{\beta} f_{T^{(1)}}\left(\Phi^{-1}\left(\frac{z}{\beta}\right)\right) D \Phi^{-1}\left(\frac{z}{\beta}\right) \mathrm{d} z .
$$

But, from

$$
\int_{0}^{\Phi^{-1}(z / \beta)} \phi_{s}\left(y_{0}\right) \mathrm{d} s=\frac{z}{\beta},
$$

we obtain, by differentiation with respect to $z$,

$$
\phi_{\Phi^{-1}(z / \beta)}\left(y_{0}\right) D \Phi^{-1}\left(\frac{z}{\beta}\right)=1
$$

and, inserting this into (4.2), it follows that $X_{T^{(1)}{ }_{-}}-x_{0}$ has the desired exponential distribution.)

We shall give a second example with $Y^{\circ}$ completely deterministic where the $c_{\gamma, \theta}$ have a simple explicit form and where the Cramér-Lundberg equation (3.6) also has a simple form. Suppose that $Y^{\circ}$ is completely deterministic and greater than 0 with $Y_{t}^{\circ}=Y_{0}^{\circ} \mathrm{e}^{a t}$ corresponding to the generator

$$
\mathcal{A}_{Y} \circ g(y)=\operatorname{ayg}^{\prime}(y) \text {; }
$$

cf. (4.1). We assume that $a \neq 0$. Next we assume that $\lambda(y)=\lambda>0$ and that

$$
\beta(y)=\beta \mathbf{1}_{[r, \infty)}(y),
$$


where $\beta>0$ and $r>0$. Then (3.5) becomes

$$
\left(\gamma \beta \mathbf{1}_{[r, \infty)}(y)-\lambda-\theta\right) c_{\gamma}(y)+a y c_{\gamma}^{\prime}(y)=\lambda,
$$

which, if $\operatorname{Re} \gamma \leq 0$, has the following unique absolutely continuous and bounded solution for $y>0$ :

$$
\begin{aligned}
& a>0: c_{\gamma, \theta}(y)=\left\{\begin{array}{lr}
-\frac{\lambda}{\lambda+\theta}+\left(\frac{y}{r}\right)^{(\lambda+\theta) / a}\left(\frac{\lambda}{\lambda+\theta}-\frac{\lambda}{\lambda+\theta-\beta \gamma}\right), & y \leq r, \\
-\frac{\lambda}{\lambda+\theta-\beta \gamma}, & y \geq r,
\end{array}\right. \\
& a<0: \quad c_{\gamma, \theta}(y)= \begin{cases}-\frac{\lambda}{\lambda+\theta}, & y \leq r, \\
-\frac{\lambda}{\lambda+\theta-\beta \gamma} & y \geq r . \\
+\left(\frac{y}{r}\right)^{(\lambda+\theta-\beta \gamma) / a}\left(\frac{\lambda}{\lambda+\theta-\beta \gamma}-\frac{\lambda}{\lambda+\theta}\right), & \end{cases}
\end{aligned}
$$

Assume from now on that the reset distribution is degenerate at $r, A=\varepsilon_{r}$. The CramérLundberg equation (3.6) is then particularly simple, viz.

$$
\begin{aligned}
R_{+}(\gamma)\left(1+\left(1-p_{U}\right) L_{-}(\gamma) \frac{\lambda}{\gamma \beta-\lambda-\theta}\right) & =-p_{U} P_{+}(\gamma) \frac{\lambda}{\gamma \beta-\lambda-\theta}, \\
R_{+}(\gamma)\left(1+\left(1-p_{U}\right) L_{-}(\gamma) \frac{-\lambda}{\lambda+\theta}\right) & =-p_{U} P_{+}(\gamma) \frac{-\lambda}{\lambda+\theta},
\end{aligned}
$$

using (4.3) for the case $a>0$ and (4.4) for the case $a<0$.

If $a>0$, this is exactly the same as (2.8) from Example 2.1 with $X$ the standard risk process with Poisson $\lambda$ arrivals of claims and constant premium rate $\beta$ !

If $a<0$, and we assume that $F_{+}$is exponential at rate $\mu>0$ and $F_{-}$is exponential (on $(-\infty, 0))$ at rate $v>0,(4.4)$ reduces to a second-order equation in $\gamma$ which, for $\theta>0$, has one root less than 0 that should be used in (3.7) and one root greater than 0 . For $\theta=0$, the two solutions are $\gamma=0$ and $\gamma_{1}=p_{U} v-\left(1-p_{U}\right) \mu$, which does not depend on $a, b$, or $r$. Thus, the ruin probability $\mathbb{P}_{x, y}(\tau<\infty)=1$ if and only if $p_{U} v-\left(1-p_{U}\right) \mu \geq 0$ if and only if $\mathbb{E} U_{n} \geq 0$, while, if $p_{U} v-\left(1-p_{U}\right) \mu<0$, we find, using (3.35), (3.36), and (3.37), that

$$
\mathbb{P}_{x, y}(\tau<\infty)=-\frac{p_{U}}{1-\left(1-p_{U}\right) v /\left(v-\gamma_{1}\right)} c_{\gamma_{1}, 0}(y) \mathrm{e}^{\gamma_{1} x},
$$

with

$$
c_{\gamma, 0}(y)= \begin{cases}-1, & y \leq r, \\ -\frac{\lambda}{\lambda-\beta \gamma}+\left(\frac{y}{r}\right)^{(\lambda-\beta \gamma) / a}\left(\frac{\lambda}{\lambda-\beta \gamma}-1\right), & y \geq r .\end{cases}
$$

Example 4.3. Suppose that $Y^{\circ}$ is a one-dimensional diffusion, i.e.

$$
\mathrm{d} Y_{t}^{\circ}=b\left(Y_{t}^{\circ}\right) \mathrm{d} t+\sigma\left(Y_{t}^{\circ}\right) \mathrm{d} B_{t},
$$


on some open, half-open, or closed interval $I$ from $l$ to $r,-\infty \leq l<r \leq \infty$. Then the domain of the generator $A_{Y}$ includes all bounded functions $g$ that are twice continuously differentiable on the interior of $I$, int $I$, with

$$
\mathcal{A}_{Y^{\circ}} g(y)=b(y) g^{\prime}(y)+\frac{1}{2} \sigma^{2}(y) g^{\prime \prime}(y)
$$

bounded and that, if either $l \in I$ or $r \in I$, satisfy the boundary conditions pertaining to a reflecting or absorbing boundary point. (The functions $b$ and $\sigma$ must be compatible with $I$ in order for the diffusion to exist, e.g. in order for $Y^{\circ}$ never to hit $l$ if $l \notin I$.)

In order to use Theorem 3.1, we must solve (see (3.5)) the second-order differential equation

$$
(\gamma \beta(y)-\lambda(y)-\theta) c_{\gamma, \theta}(y)+b(y) c_{\gamma, \theta}^{\prime}(y)+\frac{1}{2} \sigma^{2}(y) c_{\gamma, \theta}^{\prime \prime}(y)=\lambda(y), \quad y \in I,
$$

subject to the relevant boundary conditions. As a specific case, suppose that $Y^{\circ}$ is a CoxIngersoll-Ross process on the open interval $I=(0, \infty)$, i.e.

$$
\mathrm{d} Y_{t}^{\circ}=\left(a+b Y_{t}^{\circ}\right) \mathrm{d} t+\sigma \sqrt{Y_{t}^{\circ}} \mathrm{d} B_{t},
$$

where $a \in \mathbb{R}, b \in \mathbb{R}$, and $\sigma>0$ satisfying $2 a \geq \sigma^{2}$, which is the well-known necessary and sufficient condition on $(a, b, \sigma)$ that insures that the diffusion stays inside $I=(0, \infty)$ forever. Assume also that $\lambda(y) \equiv \lambda>0$ (Poisson arrival of claims) and that $\beta(y)=\beta y$ with $\beta>0$. Equation (4.5) then reduces to

$$
(\gamma \beta y-\lambda-\theta) c_{\gamma, \theta}(y)+(a+b y) c_{\gamma, \theta}^{\prime}(y)+\frac{1}{2} \sigma^{2} y c_{\gamma, \theta}^{\prime \prime}(y)=\lambda
$$

with no boundary conditions, an equation we now proceed to solve assuming that $\operatorname{Re} \gamma<0$ and $\theta>0$.

Define $\rho_{ \pm}=\rho_{ \pm}(\gamma)$ as the roots of the equation $\gamma \beta-b z+\frac{1}{2} \sigma^{2} z^{2}=0$, i.e.

$$
\rho_{ \pm}(\gamma)=\frac{1}{\sigma^{2}}\left(b \pm \sqrt{b^{2}-2 \beta \gamma \sigma^{2}}\right)
$$

where $\sqrt{b^{2}-2 \beta \gamma \sigma^{2}}$ is the square root of $b^{2}-2 \beta \gamma \sigma^{2}$ with strictly positive real part. Then $\operatorname{Re} \rho_{+}>0, \operatorname{Re} \rho_{-}<0$, and $\operatorname{Re}\left(\rho_{-} / \rho_{+}\right)<0$. Furthermore, define $\delta_{ \pm}=\delta_{ \pm}(\gamma, \theta) \in \mathbb{C}$ by

$$
\delta_{ \pm}=\frac{2}{\sigma^{2}\left(\rho_{+}-\rho_{-}\right)}\left(\lambda+\theta+b+\left(a-\sigma^{2}\right) \rho_{ \pm}\right)
$$

and, finally, define, for $0 \leq r<1$,

$$
\psi_{\gamma, \theta}(r)=(1-r)^{\delta_{+}}\left(r-\frac{\rho_{-}}{\rho_{+}}\right)^{-\delta_{-}},
$$

using the main branch of the complex logarithm function to define $\log \left(r-\rho_{-} / \rho_{+}\right)$. Then $\operatorname{Re} \delta_{+}>-1$, wherefore the integrals

$$
\Psi_{\gamma, \theta}(t)=-\int_{r}^{1} \psi_{\gamma, \theta}(u) \mathrm{d} u, \quad \tilde{\Psi}_{\gamma, \theta}(r)=-\int_{r}^{1} u \psi_{\gamma}(u) \mathrm{d} u
$$

converge absolutely and the function

$$
y \mapsto \tilde{c}_{\gamma, \theta}(y)=\int_{0}^{1} \psi_{\gamma, \theta}(t) \mathrm{e}^{-t \rho_{+} y} \mathrm{~d} t
$$


solves the second-order differential equation

$$
(\gamma \beta y-\lambda-\theta) \tilde{c}_{\gamma, \theta}(y)+(a+b y) \tilde{c}_{\gamma, \theta}^{\prime}(y)+\frac{1}{2} \sigma^{2} y \tilde{c}_{\gamma, \theta}^{\prime \prime}(y)=(\lambda+\theta) \Psi_{\gamma, \theta}(0)+a \rho_{+} \widetilde{\Psi}_{\gamma, \theta}(0),
$$

i.e. (4.6) is solved by

$$
c_{\gamma, \theta}(y)=\frac{\lambda}{(\lambda+\theta) \Psi_{\gamma, \theta}(0)+a \rho_{+} \widetilde{\Psi}_{\gamma, \theta}(0)} \int_{0}^{1} \psi_{\gamma, \theta}(u) \mathrm{e}^{-u \rho_{+} y} \mathrm{~d} u .
$$

We shall not here give the technical details for the proofs of all the claims made above, but only indicate how one argues that $\tilde{c}_{\gamma}$ solves (4.8). The left-hand side of (4.8) equals

$$
\begin{aligned}
& (\gamma \beta y-\lambda-\theta) \int_{0}^{1} \psi_{\gamma, \theta}(u) \mathrm{e}^{-u \rho_{+} y} \mathrm{~d} u-(a+b y) \int_{0}^{1} \rho_{+} u \psi_{\gamma, \theta}(u) \mathrm{e}^{-u \rho_{+} y} \mathrm{~d} u \\
& +\frac{1}{2} \sigma^{2} y \int_{0}^{1} \rho_{+}^{2} u^{2} \psi_{\gamma, \theta}(u) \mathrm{e}^{-u \rho_{+} y} \mathrm{~d} u
\end{aligned}
$$

which, using partial integration, may be written as

$$
y \int_{0}^{1} \varepsilon(u) \mathrm{e}^{-u \rho_{+} u} \mathrm{~d} u+(\lambda+\theta) \Psi_{\gamma, \theta}(0)+a \rho_{+} \widetilde{\Psi}_{\gamma, \theta}(0),
$$

where

$$
\varepsilon(u)=\left(\gamma \beta-b \rho_{+} u+\frac{1}{2} \sigma^{2} \rho_{+}^{2} u^{2}\right) \psi_{\gamma, \theta}(u)-(\lambda+\theta) \rho_{+} \Psi_{\gamma, \theta}(u)-a \rho_{+}^{2} \widetilde{\Psi}_{\gamma, \theta}(u) .
$$

We claim that $\varepsilon(u) \equiv 0$ : by differentiation,

$$
\varepsilon^{\prime}(u)=\left(\gamma \beta-b \rho_{+} u+\frac{1}{2} \sigma^{2} \rho_{+}^{2} u^{2}\right) \psi_{\gamma, \theta}^{\prime}(u)-\rho_{+}\left(\lambda+\theta+b+\left(a-\sigma^{2}\right) \rho_{+} u\right) \psi_{\gamma, \theta}(u),
$$

and the definition of $\psi_{\gamma, \theta}$ given in (4.7) fits precisely with the fact that $\varepsilon^{\prime}$ vanishes. Thus, $\varepsilon$ is constant, and since $\Psi_{\gamma, \theta}(1)=\widetilde{\Psi}_{\gamma, \theta}(1)=0$, this constant equals

$$
\lim _{u \rightarrow 1}\left(\gamma \beta-b \rho_{+} u+\frac{1}{2} \sigma^{2} \rho_{+}^{2} u^{2}\right) \psi_{\gamma, \theta}(u)=\lim _{u \rightarrow 1} \frac{1}{2} \sigma^{2}\left(\rho_{+} u-\rho_{+}\right)\left(\rho_{+} u-\rho_{-}\right) \psi_{\gamma, \theta}(u)=0,
$$

using the fact that $\rho_{+}$and $\rho_{-}$are the roots of $\gamma \beta-b z+\frac{1}{2} \sigma^{2} z^{2}=0$ together with (4.7) and $\operatorname{Re} \delta_{+}>-1$ for the last equality.

Equation (4.9) may be written as

$$
c_{\gamma, \theta}(y)=-\frac{\int_{0}^{1} \lambda \psi_{\gamma, \theta}(u) \mathrm{e}^{-u \rho_{+} y} \mathrm{~d} u}{\int_{0}^{1}\left(\lambda+\theta+a \rho_{+} u\right) \psi_{\gamma, \theta}(u) \mathrm{d} u} .
$$

From (3.4) we know that $\left|c_{\gamma, \theta}(y)\right|<1$, an inequality that is obvious upon referring to the form (4.7) of $\psi_{\gamma, \theta}$ when $\gamma \in \mathbb{R}, \gamma<0$, but which in the general case, $\gamma \in \mathbb{C}$ and $\operatorname{Re} \gamma<0$, appears surprisingly difficult to prove by analytic means!

\section{Acknowledgements}

This work was supported by the Danish Research Council for the Natural Sciences (FNU), grant number 272-06-0442, Point Process Modelling and Statistical Inference. Thanks are also due to a referee for valuable comments and for pointing out the relevance of [1]. 


\section{References}

[1] Albrecher, H. and Boxma, O. J. (2005). On the discounted penalty function in a Markov-dependent risk model. Insurance Math. Econom. 37, 650-672.

[2] Asmussen, S. (2000). Ruin Probabilities (Adv. Ser. Statist. Sci. Appl. Prob. 2). World Scientific, River Edge, NJ.

[3] Davis, M. H. A. (1993). Markov Models and Optimization (Monogr. Statist. Appl. Prob. 49). Chapman and Hall, London.

[4] Ganesh, A. Macci, C. and Torrisi, G. L. (2007). A class of risk processes with reserve-dependent premium rate: sample path large deviations and importance sampling. Queueing Systems 55, 83-94.

[5] Jacobsen, M. (2003). Martingales and the distribution of the time to ruin. Stoch. Process. Appl. 107, $29-51$.

[6] Jacobsen, M. (2005). The time to ruin for a class of Markov additive risk process with two-sided jumps. Adv. Appl. Prob. 37, 963-992.

[7] Jacobsen, M. (2005). Point Processes, Theory and Applications. Birkhäuser, Boston, MA.

[8] JaCobsen, M. And Jensen, A. T. (2007). Exit times for a class of piecewise exponential Markov processes with two-sided jumps. Stoch. Process. Appl. 117, 1330-1356.

[9] JASIULEwICZ, H. (2001). Probability of ruin with variable premium rate in a Markovian environment. Insurance Math. Econom. 29, 291-296.

[10] Møller, C. M. (1995). Stochastic differential equations for ruin probabilities. J. Appl. Prob. 32, 74-89.

[11] Paulsen, J. (2008). Ruin models with investment income. Prob. Surveys 5, 416-434.

[12] Paulsen, J. and Gjessing, H. K. (1997). Ruin theory with stochastic return on investments. Adv. Appl. Prob. 29, 965-985.

[13] Wu, R. AND WeI, L. (2004). The probability of ruin in a kind of Cox risk model with variable premium rate. Scand. Actuarial J. 2004, 121-132. 